\title{
Acción del oleaje en la estabilidad del lecho marino en la sonda de Campeche, México
}

\author{
E.G. Mendoza-Baldwin, E.E. Salazar-Carrillo² y R. Silva-Casarín \\ Grupo de Ingeniería de Costas y Puertos, Instituto de Ingeniería. UNAM, Instituto Mexicano del Petróleo ${ }^{2}$. \\ E-mail:emendozab@iingen.unam.mx
}

(recibido: agosto de 2003; aceptado: febrero de 2004)

\section{Resumen}

La seguridad de las actividades relacionadas con el litoral depende, en gran medida, del correcto funcionamiento de las estructuras de operación y protección, el diseño de las cuales, a su vez, requiere de un conocimiento lo más completo posible, tanto de las solicitaciones como del comportamiento del entorno en el que serán construidas. Como respuesta a dichas necesidades, en este trabajo se presenta la aplicación de un modelo en tres dimensiones con el que se puede representar la respuesta del lecho marino, considerando la existencia de un estrato poroso de espesor finito ante la acción de un sistema de ondas progresivas de cresta corta, el cual es de especial utilidad para el diseño y tendido de líneas submarinas.

Dentro de las bondades del modelo se cuenta con la posibilidad de variar las condiciones del tipo de suelo (cohesivo, arenoso o mixto), grado de saturación, ángulo de incidencia del oleaje y espesor del estrato poroso.

El modelo tridimensional es alimentado con datos de oleaje y tipo de suelo de la bahía de Campeche, México, con los que se analizan las distribuciones verticales de los esfuerzos y la presión de poro inducidos.

Finalmente, se realiza un estudio de inestabilidad del lecho marino para la misma bahía, comparando los resultados del modelo con los de sondeos en campo.

Descriptores: interacción oleaje-suelo, medio poroso, esfuerzos inducidos por oleaje, inestabilidad del fondo.

\footnotetext{
Abstract

Among the phenomena that must be taken into account while designing and constructing coastal structures, the wave-induced soil response has become a very important issue. One reason for this growing interest is that many structures have been reportedly failed because of seabed instability rather than design or construction deficiencies.

In this paper we present the application to Sonda de Campeche. Mexico of an analytical model for a porous seabed of finite thickness, h, subject to a 3D short crested wave system. This exact solution gives explicit expressions for the pore pressure and effective stresses under
} 
Acción del oleaje en la estabilidad del lecho marino en la sonda de Campeche, México

DOI:http://dx.doi.org/10.22201/fi.25940732e.2004.05n4.017

the following assumptions:

Horizontal seabed

Homogeneous, unsaturated and hydraulically anisotropic soil.

The soil skeleton and the pore fluid are uniformly compressible.

The soil skeleton obeys Hooke's law, implying linear, reversible and non-retarded mechanical properties.

The flow in the porous bed is governed by Darcy's law.

In this paper the porous bed is to be considered cohesive; nevertheless the original model can be applied to sandy or mixed soils.

The governing equations are obtained from Biot's consolidation theory ( and Verruijt's storage equation (combined with equilibrium and momentum equations.

Keywords: soil-wave interaction, porous media, wave induced stresses, seabed instability.

\section{Introducción}

La estabilidad de las estructuras apoyadas sobre el lecho marino depende, además del adecuado conocimiento de las solicitaciones físicas y de los diseños geométrico y mecánico, del análisis del comportamiento del suelo en el que se van a cimentar. Despreciar el efecto de la posible inestabilidad del lecho ha sido la razón de falla de numerosas estructuras.

En México, la estabilidad de las construcciones empleadas para la extracción y transporte de hidrocarburos cobra especial atención, es decir, las líneas submarinas. Tan solo en la bahía de Campeche se tienen más de $2500 \mathrm{~km}$ de tubería dispuesta, sobre o enterrada en el fondo marino, el cual al estar compuesto principalmente de material cohesivo, es susceptible a inestabilizarse e incluso llegar a la licuefacción. Como ejemplo, en el año 1995, con la ocurrencia de los huracanes Pauline y Roxanne, se encontraron líneas de conducción que habían sufrido desplazamientos del orden de cientos de metros, debido principalmente a que al inestabilizarse el fondo marino, la tubería queda expuesta directamente a las acciones de las fuerzas ejercidas por el oleaje.
Tradicionalmente, para abordar este problema en el diseño, se han empleado modelos simplificados, los cuales consideran al suelo como un medio linealmente elástico de una fase, como los desarrollados por Moshagen y Torum (1975); Mallard y Dalrympe (1977). Esta primera suposición carece de validez debido a que el oleaje induce, a través de los poros, una presión en exceso a la estructura del sólido, con lo que el sistema deja de ser de una sola fase (Jeng, 1997). Adicionalmente, es conocido que cuando dicha presión en exceso sobrepasa un cierto umbral, se presenta la falla del lecho marino, de ahí la necesidad de contar con un modelo más completo. La principal ventaja de utilizar la teoría de Biot sobre aquellos modelos basados en aproximaciones de tipo Darcy es que permiten el estudio de cualquier tipo de suelo.

Es en este sentido que el presente trabajo estudia específicamente el comportamiento del material cohesivo asociado al clima marítimo, a la vez que analiza la posibilidad de falla del estrato ante diversas condiciones de oleaje; logrando así localizar, en el perfil vertical, las zonas susceptibles de licuefacción. El modelo matemático con el que se simula la respuesta de la masa de suelo tiene como base 
el modelo en tres dimensiones, propuesto por Hsu y Jeng (1994), con la ventaja de que la aplicación aquí utilizada puede emplearse para el estudio de un mayor rango de casos, la cual se presenta a continuación.

\section{Descripción del modelo}

\section{Antecedentes}

Numerosos investigadores han estudiado el comportamiento del fondo marino inducido por el oleaje, hallando soluciones analíticas basadas en diversas simplificaciones relacionadas con la rigidez relativa del suelo y del fluido dentro de los poros, así como con características de la masa de suelo como permeabilidad, anisotropía y grado de saturación. En estos términos se distinguen principalmente tres tipos de aproximación que se traducen en tres ecuaciones de gobierno, la ecuación de Laplace, la ecuación de difusión y la consolidación de Biot.

La primera aproximación consiste en suponer que el suelo es poroso y rígido, además de considerar que el fluido en la estructura del suelo es incompresible, condiciones para las que la ecuación que describe el exceso de presión de poro inducido por el oleaje es la de Laplace. Uno de los primeros trabajos en esta dirección es el de Putnam (1949) quien presentó un modelo simple para suelos isotrópicos porosos con ancho finito y concluyendo que la distribución de la presión en el lecho es función sólo de las características del oleaje y no del material del fondo. Sin embargo Liu (1977) al comparar los mo delos para ancho de estrato infinito con los de ancho finito, encontró que dicha distribución depende también de la permeabilidad y del ancho del estrato en menor grado. Trabajos posteriores refinaron la simulación incluyendo el equilibrio de esfuerzos y los términos inerciales en el suelo (Dawson, 1978). Más adelante Liu y Dalrymple (1984) detectaron que el decaimiento espacial en la distribución de presión depende de la permeabilidad del suelo y de la profundidad cuando el número de onda se mantiene aproximadamente constante.

En la segunda aproximación, gobernada por la ecuación de difusión o de conducción del calor, se acepta que el fluido dentro del medio poroso es compresible y la estructura del suelo es no deformable. Estas hipótesis fueron propuestas inicialmente por Nakamura et al. (1973) quienes obtuvieron resultados acordes a los estimados con la ecuación de Laplace; sin embargo, sus mediciones de laboratorio presentaban extrañas discontinuidades en la distribución de la presión cerca del fondo, las cuales, según Yamamoto (1977), se debían a que las olas que generaron (Nakamura, et al. 1973) eran muy peraltadas y ocasionaban la licuefacción del material. Por su parte, Moshagen y Torum (1975), desarrollaron un modelo que considera compresible al fluido e incompresible al material del fondo, lo que de acuerdo con Prevost y Hughes (1980) no representa la realidad, por lo que desarrollaron un modelo para fluido incompresible y suelo compresible. Esta suposición los condujo a la misma solución que para el caso de suelo y fluido incompresibles.

La desventaja de las aproximaciones hasta ahora mencionadas es que sólo proveen información sobre la distribución de la presión y no sobre los esfuerzos efectivos ni los desplazamientos del suelo (Yamamoto, 1983).

La tercera aproximación está basada en la teoría de consolidación de Biot (1941) y la ecuación de almacenamiento (Verruijt, 1969). En este caso, tanto el fluido como el medio 
poroso se aceptan compresibles bajo la acción de un sistema de ondas progresivas. Las primeras investigaciones bajo este supuesto fueron las realizadas por Madsen (1978) y Yamamoto et al. (1978) quienes desarrollaron soluciones para suelos anisotrópicos e isotrópicos, respectivamente. Otros autores que han estudiado el fenómeno con estas hipótesis son Mei y Foda (1981), quienes realizaron una aproximación a la capa límite. Por su parte, Prevost y Hughes (1980) y Gatmiri (1990), obtuvieron soluciones numéricas para geometrías complejas del fondo marino con oleaje progresivo en dos dimensiones.

Finalmente, Hsu et al. (1993) presentaron por primera vez una solución analítica en tres dimensiones para la respuesta de un suelo de espesor infinito debido al oleaje inducido. Más tarde Hsu y Jeng (1994) ampliaron su trabajo para el caso de un estrato con espesor finito, solución que es empleada en el presente estudio.

\section{Hipótesis generales}

El sistema en estudio está conformado por un suelo de espesor $h$ conocido, el cual está sometido a fuerzas inducidas por ondas de cresta corta, con ángulo de incidencia $\theta$, que son reflejadas por una estructura vertical, empotrada hasta una profundidad infinita, lo que genera oleaje reflejado hacia el interior del sistema. En la figura 1, tomada de Hsu y Jeng (1994), se muestra un esquema del fenómeno.

Las crestas resultantes se propagan en sentido paralelo a la estructura reflejante (eje $x$ ), el eje $y$ es normal al muro y el eje $z$ es positivo hacia arriba, a partir del nivel medio del mar para el problema de propagación de oleaje y de la frontera agua-suelo; para la interacción oleaje-suelo.

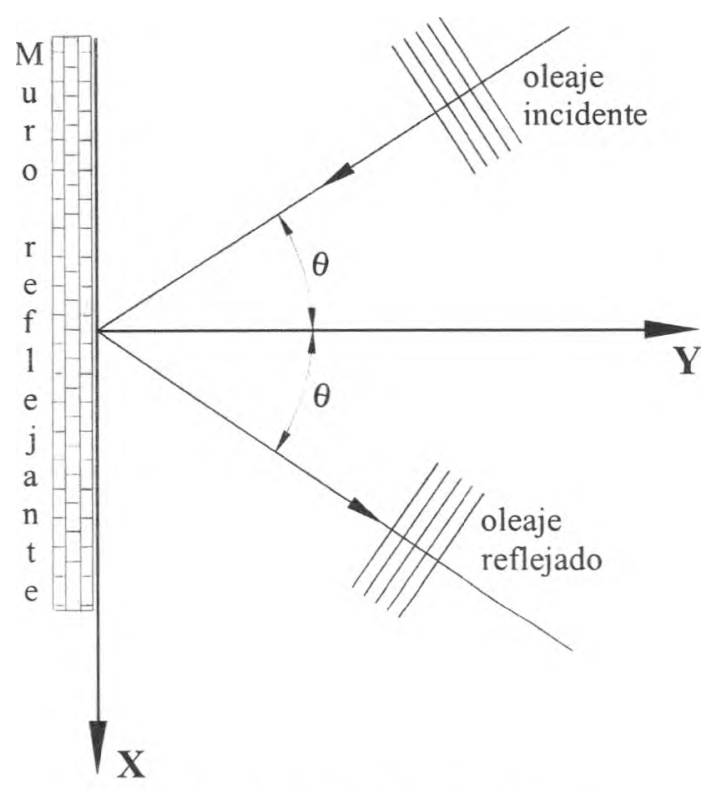

VISTA EN PLANTA

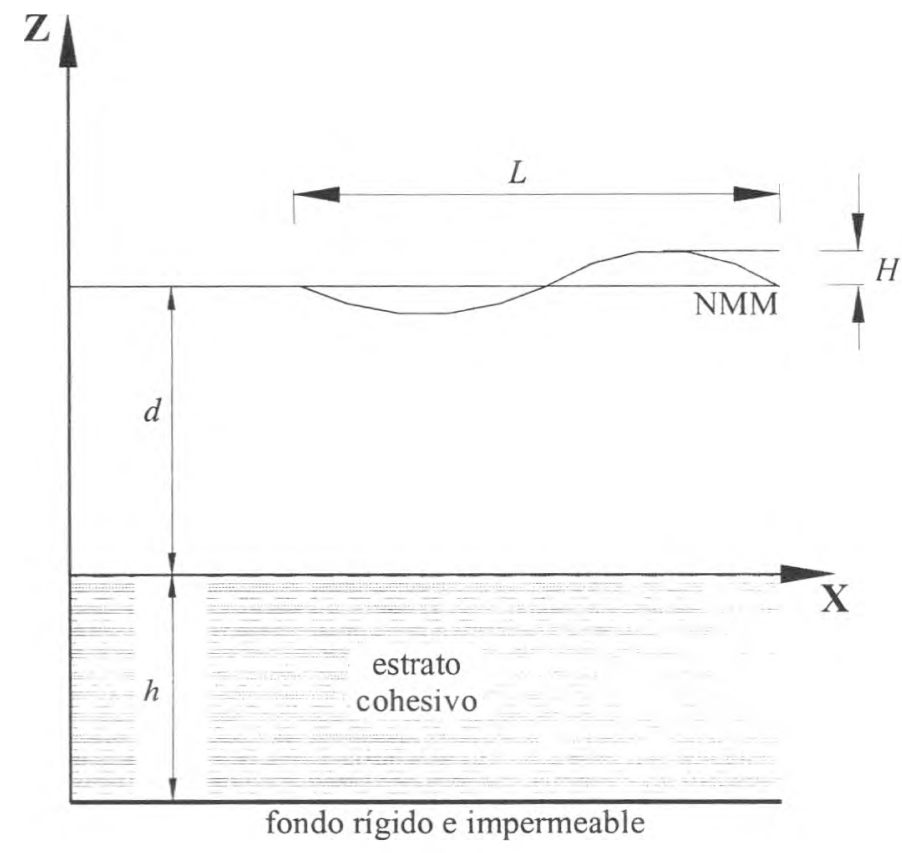

PERFIL

Figura 1. Croquis de un sistema de ondas progresivas de cresta corta 


\section{Hipótesis del medio}

El medio sobre el que se propagan las ondas es poroso y horizontal.

Por lo que respecta a sus características físicas se acepta que:

El suelo es homogéneo y no saturado, con espesor conocido e hidráulicamente anisotrópico.

La estructura del suelo y el fluido en los poros son uniformemente compresibles.

La estructura del suelo obedece la ley de Hooke, lo que implica un comportamiento mecánico lineal, reversible y no retardado.

El flujo del fluido a través del medio poroso está gobernado por la ley de Darcy.

Para los alcances de este trabajo sólo se considera suelo cohesivo, aunque el modelo original es útil, tanto para suelos arenosos como para mixtos (Salazar, 2001).

\section{Solución analítica}

A partir de la teoría de consolidación de Biot, referente a la ecuación de almacenamiento de Verruijt y considerando que el lecho marino es poroso y tiene diferentes permeabilidades en las direcciones $x, y$ y $z$, Hsu y Jeng (1994), desarrollaron la solución analítica que se presenta en las ecuaciones (1) a (4). El procedimiento que utilizaron consistió en proponer una solución que incluyera las componentes incidente y reflejada del oleaje, la cual construyeron utilizando el principio de superposición. De esta solución tomaron por separado cada componente y las combinaron con las ecuaciones de esfuerzo-deformación (ley de Hooke), de tal suerte que al resolver para cada dirección obtuvieron las expresiones correspondientes a los desplazamientos y la presión de poro para un estrato de suelo no saturado, hidráulicamente anisotrópico y de espesor finito en tres dimensiones, las cuales se presentan a continuación:

$$
\begin{gathered}
\xi=\frac{i m p_{0}}{2 G k}\left\{\left(C_{1}+C_{2} k z\right) e^{k z}+\left(C_{3}+C_{4} k z\right) e^{-k z}+k^{2} C_{5} e^{\delta z}+k^{2} C_{6} e^{-\delta z}\right\} \cos \left[(n k y) e^{i(m k x-\omega t)}\right] \\
\zeta=\frac{-n p_{0}}{2 G k}\left\{\left(C_{1}+C_{2} k z\right) e^{k z}+\left(C_{3}+C_{4} k z\right) e^{-k z}+k^{2} C_{5} e^{\delta z}+k^{2} C_{6} e^{-\delta z}\right\} \operatorname{sen}\left[(n k y) e^{i(m k x-\omega t)}\right] \\
X=\frac{P_{0}}{2 G k}\left\{\left(C_{1}-(1+2 \lambda-k z) C_{2}\right) e^{k z}-\left(C_{3}+(1+2 \lambda+k z) C_{4}\right) e^{-k z}+k \delta\left[C_{5} e^{\delta z}-C_{6} e^{-\delta z}\right]\right\} \cos \left[(n k y) e^{i(m k x-\omega t)}\right] \\
P=\frac{p_{0}}{(1-2 \mu)}\left\{(1-\lambda-2 \mu)\left(C_{2} e^{k z}-C_{4} e^{-k z}\right)+(1-\mu)\left(\delta^{2}-k^{2}\right)\left[C_{5} e^{\delta z}+C_{6} e^{-\delta z}\right]\right\} \cos \left[(n k y) e^{i(m k x-\omega t)}\right]
\end{gathered}
$$

Donde el parámetro $\lambda$ se define como: 
Acción del oleaje en la estabilidad del lecho marino en la sonda de Campeche, México DOI:http://dx.doi.org/10.22201/fi.25940732e.2004.05n4.017

$$
\lambda=\frac{(1-2 \mu)\left\{k^{2}\left[1-\frac{k_{x}}{k_{z}} m^{2}-\frac{k_{y}}{k_{z}} n^{2}\right]+\frac{i \omega \gamma_{\omega}}{k_{z}} n^{\prime} \beta\right\}}{k^{2}\left[1-\frac{k_{x}}{k_{z}} m^{2}-\frac{k_{y}}{k_{z}} n^{2}\right]+\frac{i \omega \gamma_{\omega}}{k_{z}}\left[n^{\prime} \beta+\frac{(1-2 \mu)}{G}\right]}
$$

El caso específico cuando $\lambda=0$ corresponde a un suelo completamente saturado.

Conocidos los desplazamientos, los esfuerzos efectivos normales y cortantes resultan, respectivamente:

$$
\begin{gathered}
\sigma_{x}^{\prime}=-p_{0}\left\{\left[m^{2}\left(C_{1}+C_{2} k z\right)+\frac{2 \mu \lambda}{1-2 \mu} C_{2}\right] e^{k z z}+\left[m^{2}\left(C_{3}+C_{4} k z\right)-\frac{2 \mu \lambda}{1-2 \mu} C_{4}\right] e^{-k z}+\left[k^{2} m^{2}-\frac{\mu\left(\delta^{2}-k^{2}\right.}{1-2 \mu}\right]\left(C_{5} e^{\delta z}+C_{6} e^{-\delta z}\right)\right\} \\
\cos \left[(n k y) e^{i(m k x-\omega t)}\right] \\
\sigma_{y}^{\prime}=-p_{0}\left\{\left[n^{2}\left(C_{1}+C_{2} k z\right)+\frac{2 \mu \lambda}{1-2 \mu} C_{2}\right] e^{k z}+\left[n^{2}\left(C_{3}+C_{4} k z\right)-\frac{2 \mu \lambda}{1-2 \mu} C_{4}\right] e^{-k z}+\left[k^{2} n^{2}-\frac{\mu\left(\delta^{2}-k^{2}\right)}{1-2 \mu}\right]\left(C_{5} e^{\delta z}+C_{6} e^{-\delta z}\right)\right\} \\
\sigma_{z}^{\prime}=p_{0}\left\{\left[\left(C_{1}+C_{2} k z\right)-\frac{2 \lambda(1-\mu)}{1-2 \mu} C_{2}\right] e^{k z}+\left[\left(C_{3}+C_{4} k z\right)+\frac{2 \lambda(1-\mu)}{1-2 \mu} C_{4}\right] e^{-k z}\right. \\
\left.\left.+\frac{1}{(1-2 \mu)}\left[\delta^{2}(1-\mu)-k^{2} \mu\right]\left(C_{5} e^{\delta z}+C_{6} e^{-\delta z}\right)\right\} \cos \mid(n k y) e^{i(m k x-\omega t)}\right] \\
\left.\tau_{x z}^{\prime}=i m p_{0}\left\{\left[C_{1}+(k z-\lambda) C_{2}\right] e^{k z}-\left[C_{3}+(k z+\lambda) C_{4}\right] e^{-k z}+k \delta\left(C_{5} e^{\delta z}-C_{6} e^{-\delta z}\right)\right\} \cos \mid(n k y) e^{i(m k x-\omega t)}\right] \\
\tau_{y z}^{\prime}=-n p_{0}\left\{\mid C_{1}+(k z-\lambda) C_{2}\right] e^{k z}-\left[C_{3}+(k z+\lambda) C_{4} \mid e^{-k z}+k \delta\left(C_{5} e^{\delta z}-C_{6} e^{-\delta z}\right)\right\} \operatorname{sen}\left[(n k y) e^{i(m k x-\omega t)}\right] \\
\tau_{x y}^{\prime}=-i m n p_{0}\left\{\left[C_{1}+C_{2} k z\right] e^{k z}+\left[C_{3}+C_{4} k z\right] e^{-k z}+k^{2}\left(C_{5} e^{\delta z}+C_{6} e^{-\delta z}\right)\right\} \operatorname{sen}\left[(n k y) e^{i(m k x-\omega t)}\right]
\end{gathered}
$$

Los coeficientes $\mathrm{C}_{1}$ a $\mathrm{C}_{6}$ se calculan a partir de

$$
\mathrm{C}_{i}=\frac{\mathrm{D}_{i}}{\mathrm{D}_{0}} \quad i=1 \ldots 6
$$

donde:

$D_{j}=C_{i 0}+C_{j 1} e^{-2 k \hbar}+C_{j 2} e^{-(k+\delta) h}+C_{j 3} e^{-4 k h}+C_{j 4} e^{-2 \delta \hbar}+C_{i 5} e^{-2(k+\delta) h}+C_{j 6} e^{-(3 k+\delta) h}+C_{j 7} e^{-(4 k+2 \delta) h} \quad j=0 \ldots 6$ 
los cuales han sido completamente detallados por Hsu y Jeng (1994).

La validación de la solución analítica presentada en las ecuaciones (1) a (11) la realizaron Hsu et al. (1993), simplificando el problema al caso en dos dimensiones y presentando excelentes resultados.

\section{Aplicación del modelo a la sonda de Campeche, México}

En este trabajo se realiza la aplicación de la solución analítica en tres dimensiones. Para ello se han tomado como base las características, tanto del oleaje como del material cohesivo que se presentan en la bahía de Campeche, México, los cuales se muestran en la tabla 1. (Bowles, 1997).

Tabla 1. Datos de oleaje y del estrato cohesivo en la bahía de Campeche, México

\begin{tabular}{cc}
\hline Período 7 & $12 \mathrm{~s}$ \\
Profundidad $(d)$ & $10 \mathrm{~m}$ \\
Ángulo de incidencia $(\theta)$ & $0^{\circ}-90^{\circ}$ \\
\hline
\end{tabular}

continúa...
Tabla 1. Datos de oleaje y del estrato cohesivo en la bahía de Campeche, México (continuación...)

\begin{tabular}{cc}
\hline Relación de Poisson $(\mu)$ & 0.45 \\
Porosidad $\left(n^{\prime}\right)$ & 0.2 \\
Módulo cortante $(G)$ & $10^{8} \mathrm{~N} / \mathrm{m}^{2}$ \\
Permeabilidad $(K)$ & $6 \times 10^{-7} \mathrm{~m} / \mathrm{s}$ \\
Espesor del suelo $(\mathrm{h})$ & $h=0.5 \mathrm{~L}$ \\
Grado de saturación $(\mathrm{Sr})$ & 1 \\
\hline
\end{tabular}

A continuación se presentan los resultados que ofrece el modelo para diferentes condiciones de oleaje y características del suelo, considerando el caso de altura de ola unitaria.

\section{Variación del ángulo de incidencia}

En una primera aproximación al fenómeno, se decidió analizar la distribución vertical de los esfuerzos y la presión de poro, inducidos sobre la masa del suelo para diferentes ángulos de incidencia y para el caso de espesor del estrato cohesivo, igual a la mitad de la longitud de onda. Se encontró que para dichas condiciones, tanto la presión de poro, $\mathrm{P}$ como el esfuerzo normal, $\sigma^{\prime}{ }_{z}$ presentan una distri-

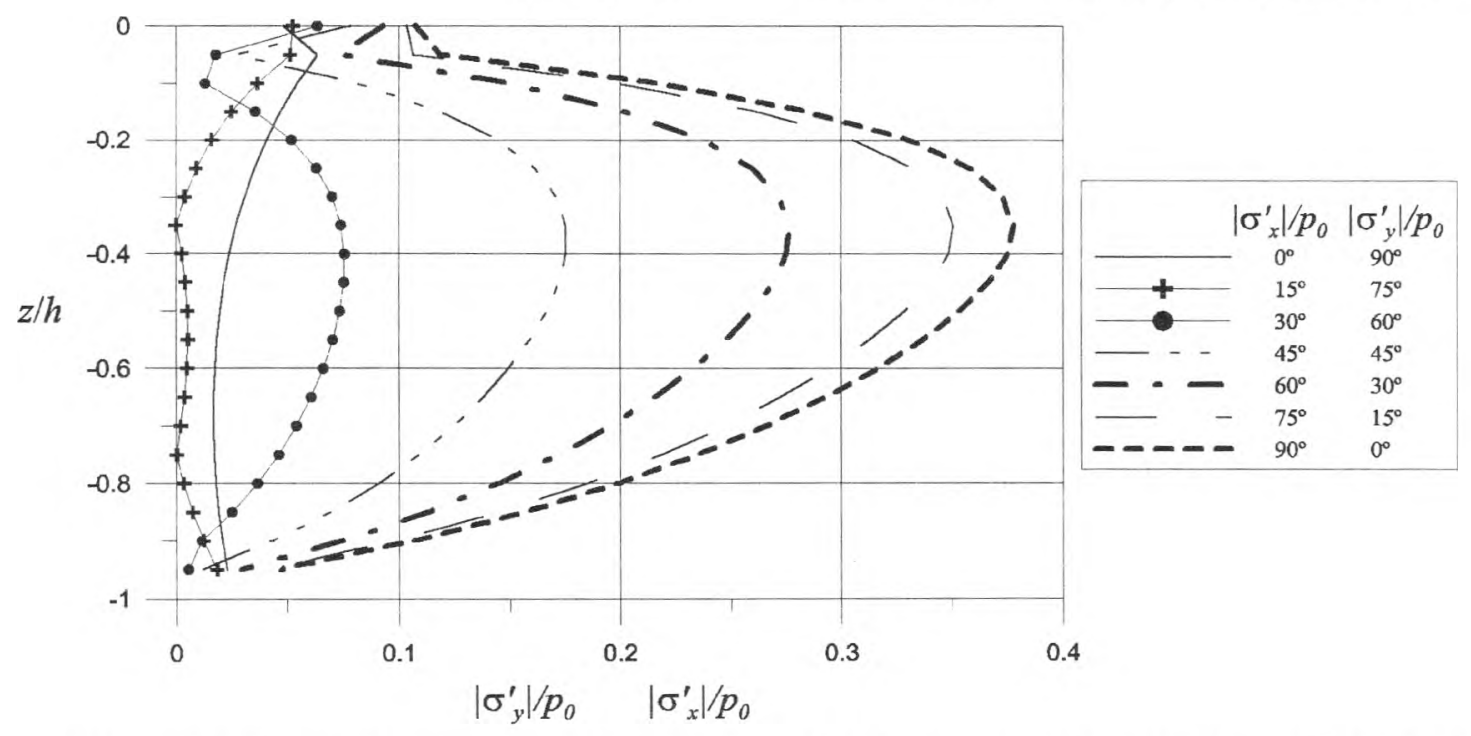

Figura 2. Distribución de los esfuerzos normales $\sigma_{y}^{\prime} \mid / p_{0}$ y $\left|\sigma^{\prime}{ }_{x}\right| / p_{0}$ para diferentes ángulos de incidencia $y$ $h=0.5 \mathrm{~L}$ 
bución idéntica para cualquier ángulo de incidencia. Los demás resultados se muestran en la figura 2.

Es importante notar que dada la anisotropía del material y el comportamiento sinusoidal del oleaje, los esfuerzos $\sigma_{x}^{\prime}$ y $\sigma_{y}^{\prime}$ coinciden para ángulos de incidencia com- plementarios. Lo mismo ocurre en el caso de los esfuerzos cortantes $\tau_{z x}$ y $\tau_{z y}$, como se muestra en la figura 3.

De igual forma, el esfuerzo cortante horizontal $\tau_{x y}$ repite sus valores para ángulos complementarios (Figura 4).

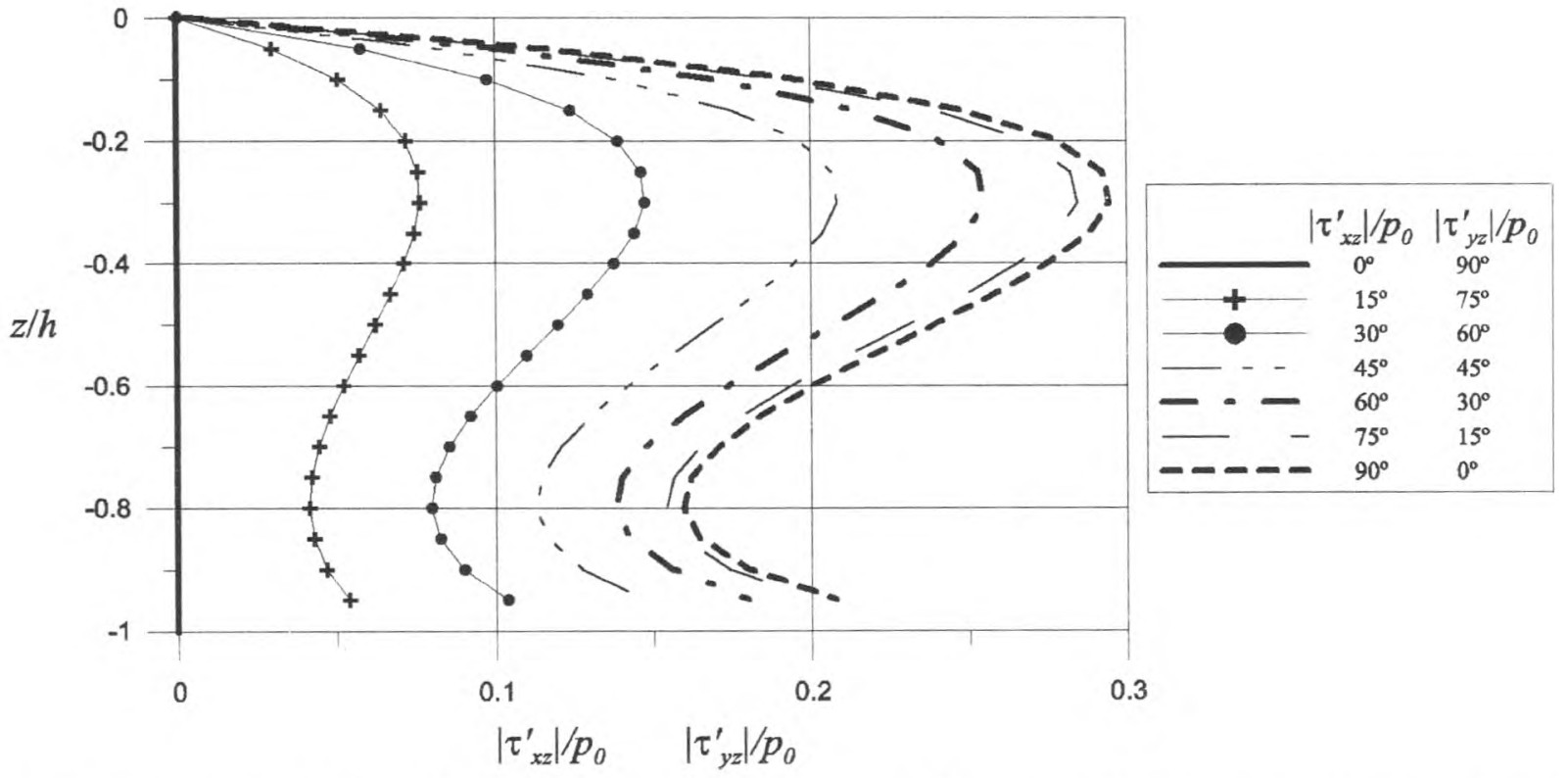

Figura 3. Distribución de los esfuerzos cortantes $\left|\tau_{z x}\right| / p_{0}$ y $\left|\tau_{z y}\right| / p_{0}$ para diferentes ángulos de incidencia y $h=0.5 \mathrm{~L}$

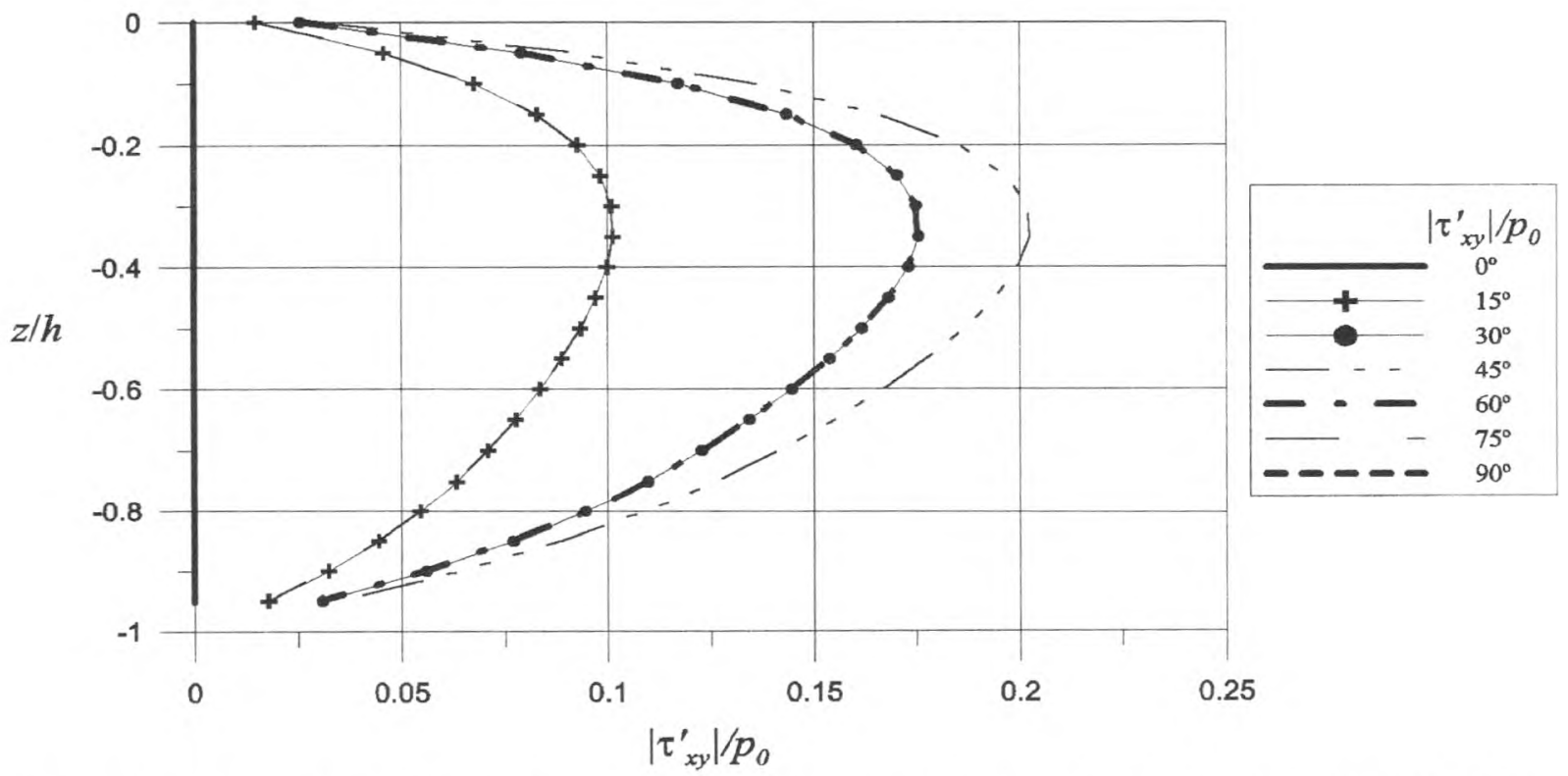

Figura 4. Distribución del esfuerzo cortante $\left|\tau_{x y}\right| / p_{0}$ para diferentes ángulos de incidencia y $h=0.5 \mathrm{~L}$ 
E.G. Mendoza-Baldwin, E.E. Salazar-Carrillo y R. Silva-Casarín

DOI:http://dx.doi.org/10.22201/fi.25940732e.2004.05n4.017

\section{Variación del espesor del estrato cohesivo}

Empleando los datos de la tabla 2 se realizó la comparación de la forma de la distribución de los esfuerzos y la presión de poro de tres espesores del estrato, $h=0.1 L, h=0.5 L$, y $h$ infinito.

Tabla 2. Datos de oleaje y del estrato cohesivo en la bahía de Campeche. México

\begin{tabular}{cc}
\hline Período $(T)$ & $12 \mathrm{~s}$ \\
Profundidad $(d)$ & $30 \mathrm{~m}$ \\
Ángulo de incidencia $(\theta)$ & $45^{\circ}$ \\
Relación de Poisson $(\mu)$ & 0.45 \\
Porosidad $\left(n^{\prime}\right)$ & 0.2 \\
Módulo cortante $(G)$ & $10^{8} \mathrm{~N} / \mathrm{m}^{2}$ \\
Permeabilidad $(K)$ & $6 \times 10^{-7} \mathrm{~m} / \mathrm{s}$ \\
Espesor del suelo $(h)$ & $h=0.1 \mathrm{~L}, 0.5 \mathrm{~L} \mathrm{y} \mathrm{h}$ infinito \\
Grado de saturación $(\mathrm{S} r)$ & 1 \\
\hline
\end{tabular}

A continuación, en las figuras 5 a 9 , se presentan los resultados obtenidos.

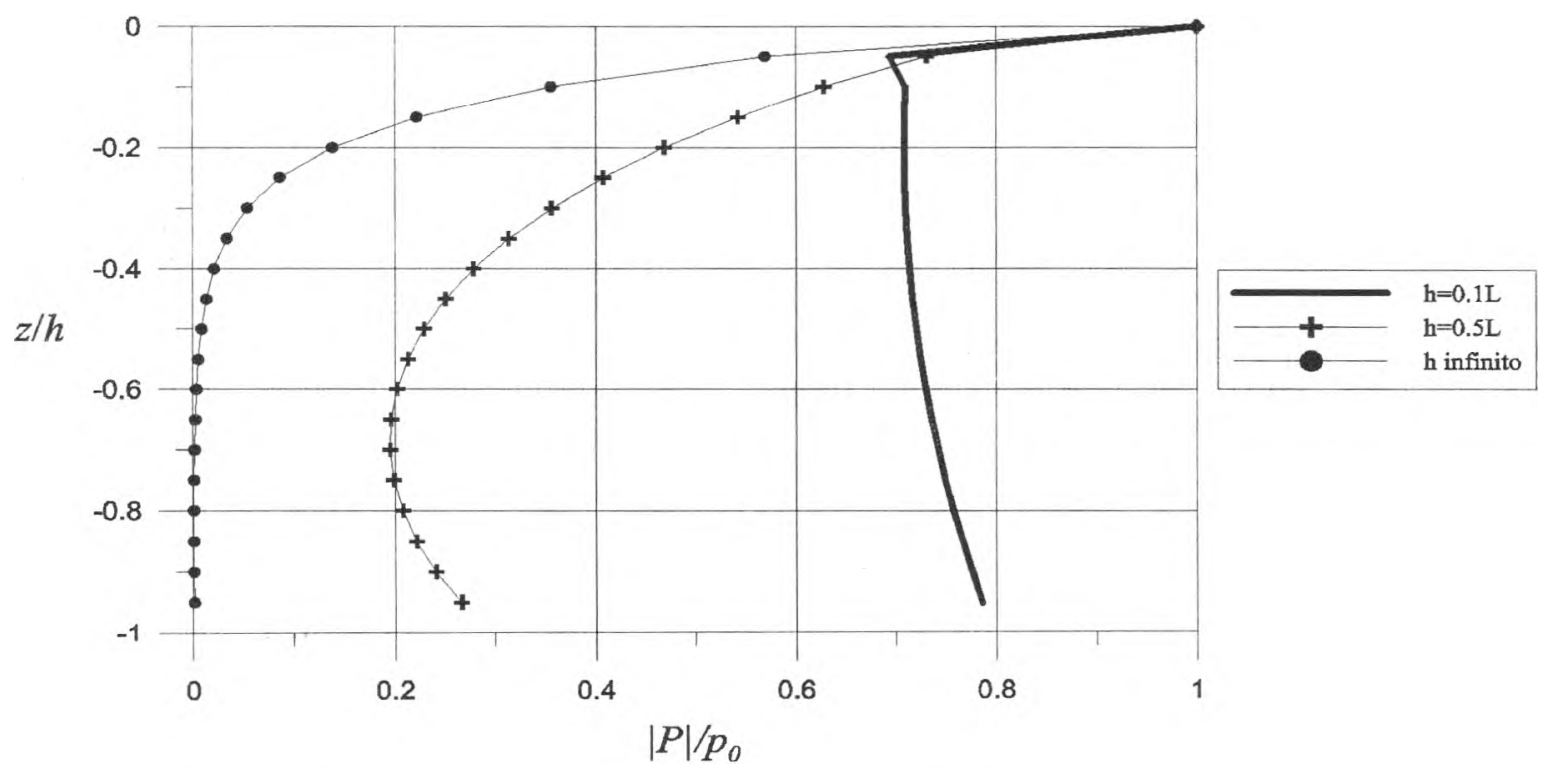

Figura 5. Distribución de la presión de poro $|\mathrm{P}| / p_{0}$ para diferentes espesores del medio poroso 
Acción del oleaje en la estabilidad del lecho marino en la sonda de Campeche. México DOI:http://dx.doi.org/10.22201/fi.25940732e.2004.05n4.017

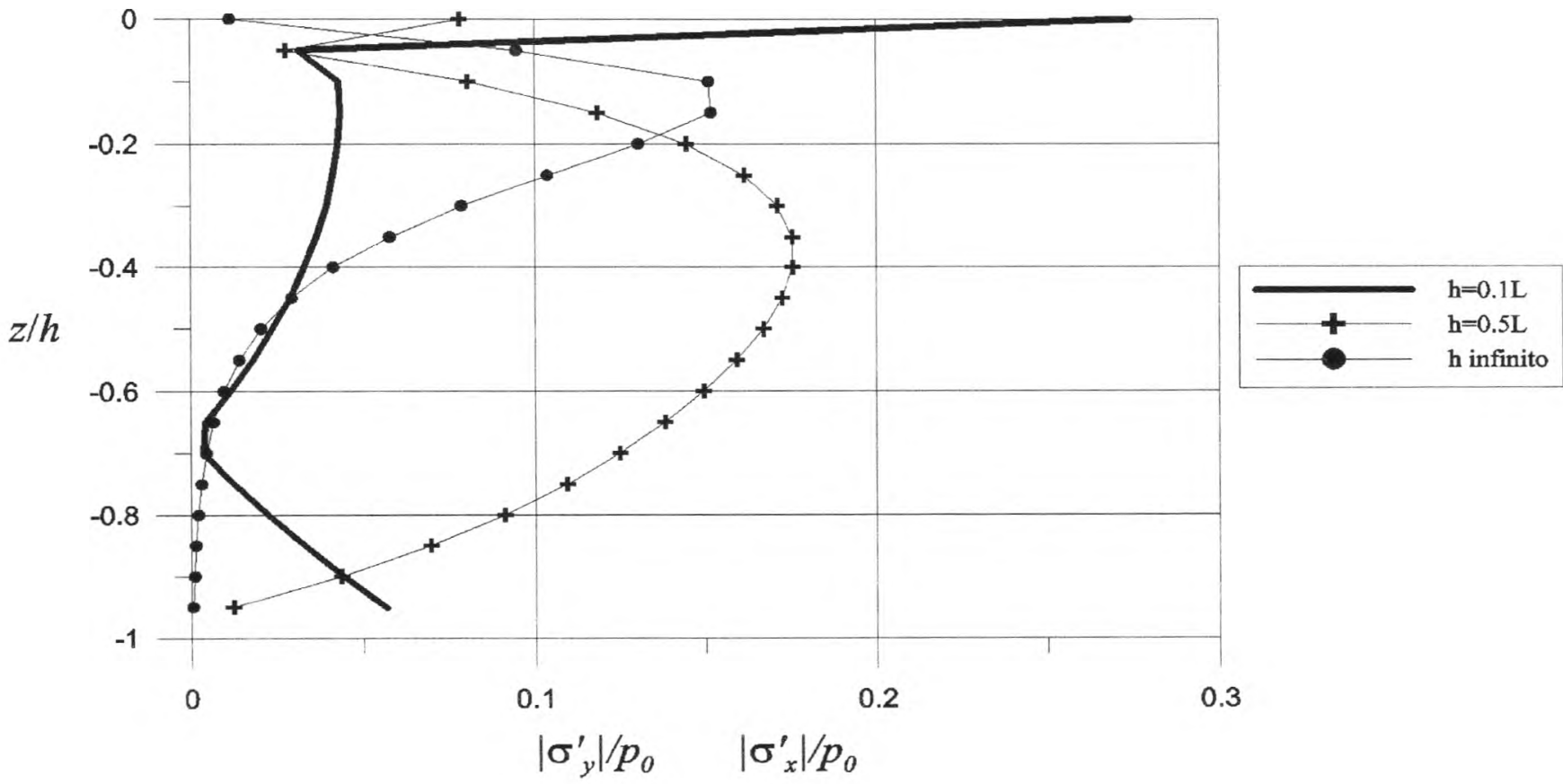

Figura 6. Distribución de los esfuerzos normales $\left|\sigma_{y}^{\prime}\right| / p_{0}$ y $\left|\sigma^{\prime}{ }_{x}\right| / p_{0}$ para diferentes espesores del medio poroso

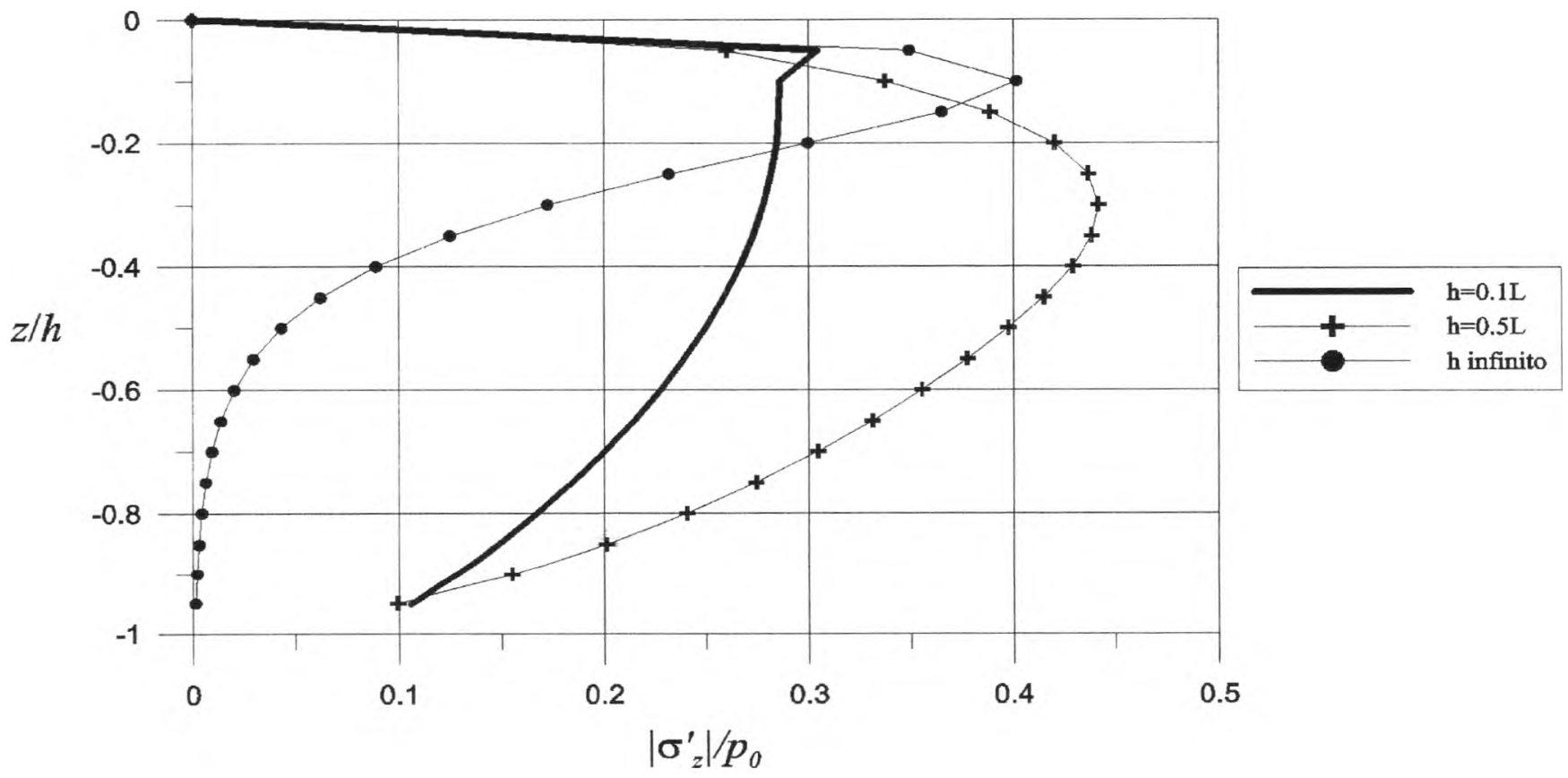

Figura 7. Distribución de los esfuerzos normales $\left|\sigma^{\prime}{ }_{z}\right| / p_{0}$ para diferentes espesores del medio poroso 
E.G. Mendoza-Baldwin, E.E. Salazar-Carrillo y R. Silva-Casarín

DOI:http://dx.doi.org/10.22201/fi.25940732e.2004.05n4.017

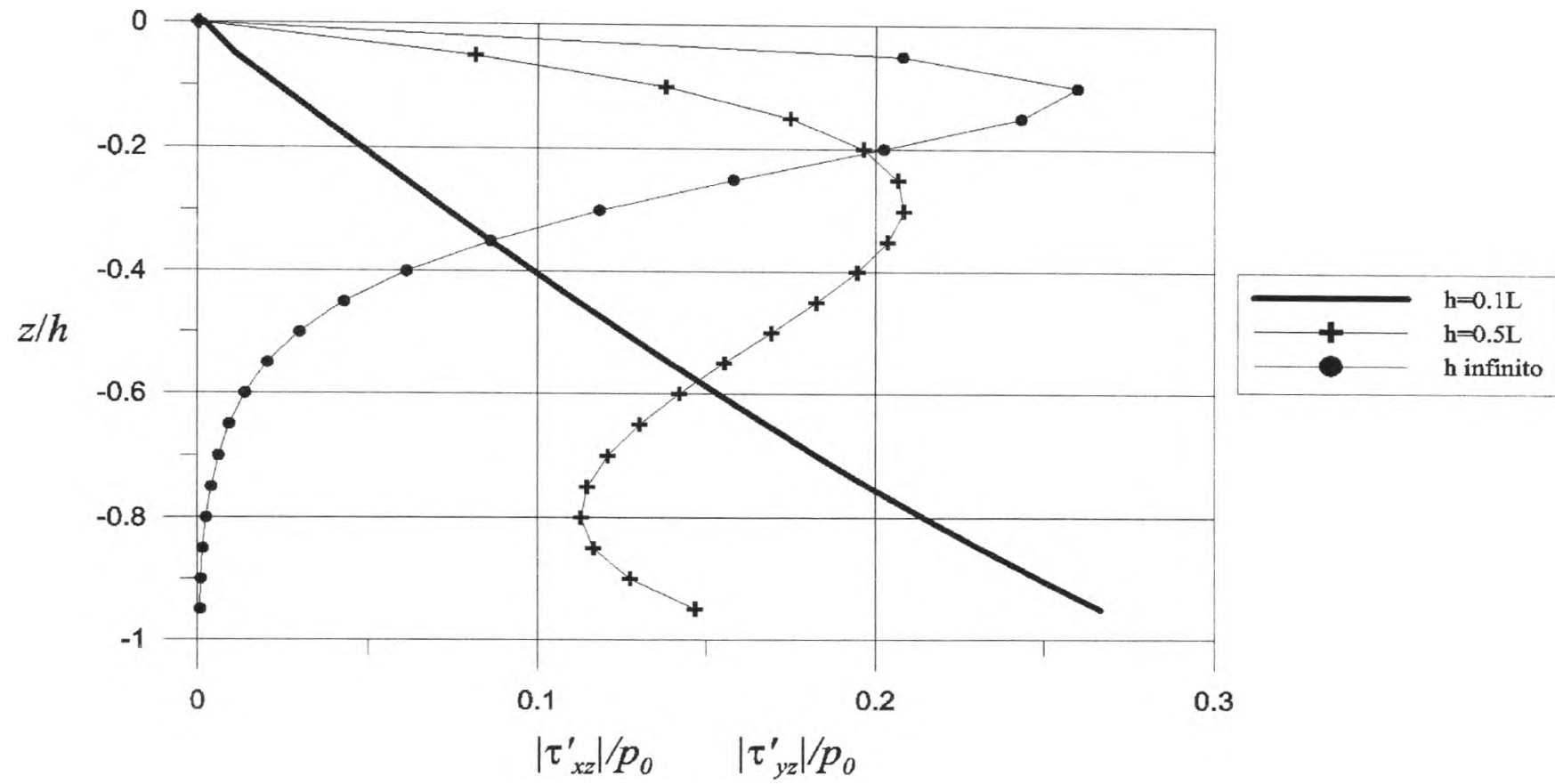

Figura 8. Distribución de los esfuerzos cortantes $\left|\tau_{z x}\right| / p_{0}$ y $\left|\tau_{z y}\right| / p_{0}$ para diferentes espesores del medio poroso

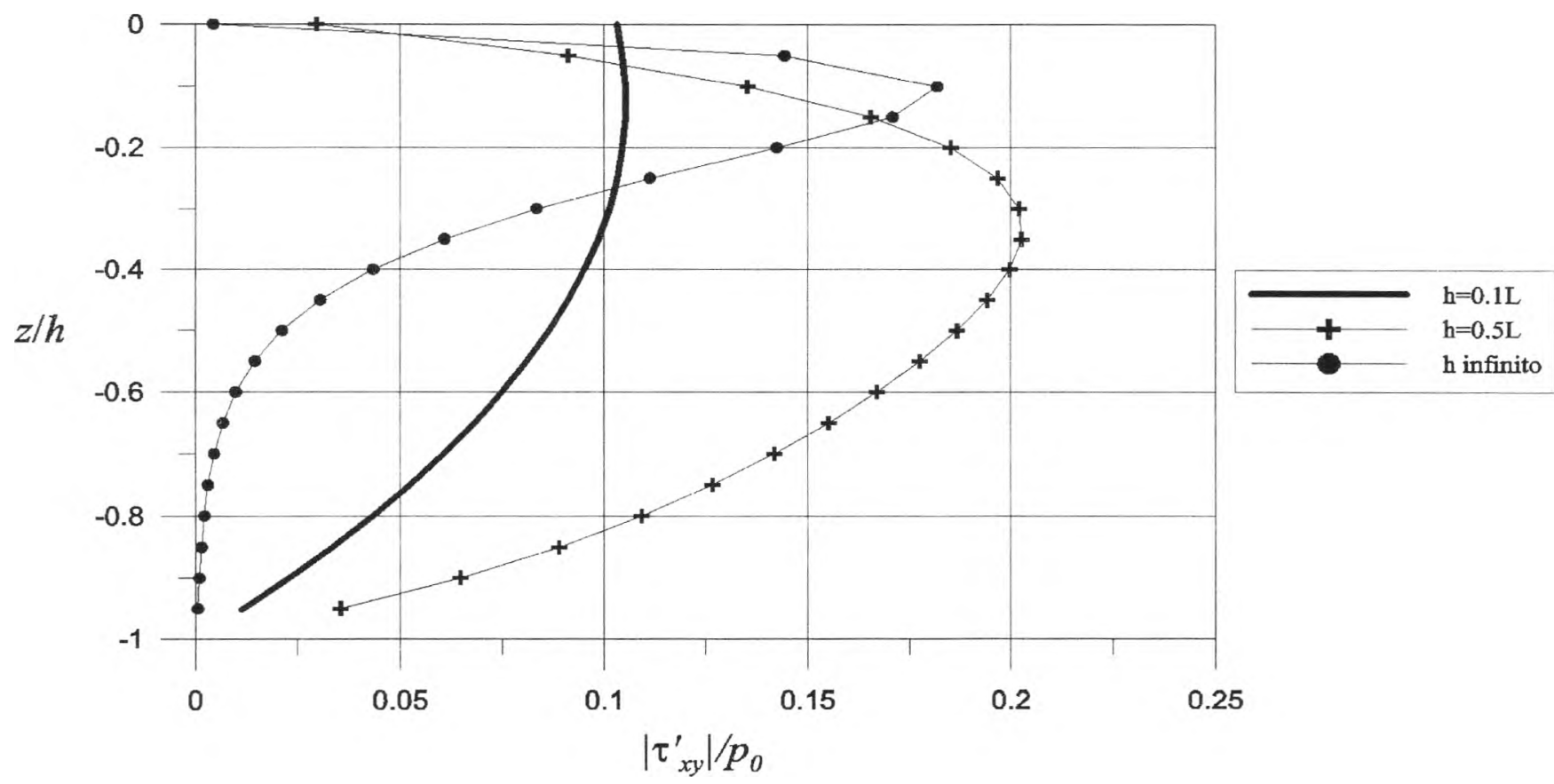

Figura 9. Distribución del esfuerzo cortante $\left|\tau_{x y}\right| / p_{0}$ para diferentes espesores del medio poroso 
Es de notar que la forma de la distribución, tanto de los esfuerzos como de la presión de poro, tiende a una curva similar a medida que aumenta el espesor del estrato. Por otro lado, el valor máximo de los esfuerzos pareciera presentarse muy cerca de la interacción aguasuelo cohesivo (aproximadamente en $-0.1 z / h$ ), lo cual resulta de especial interés en el estudio de la inestabilidad del fondo marino.

\section{Variación del grado de saturación}

Finalmente y ante la posibilidad de que el fondo cohesivo no se encuentre totalmente saturado, se realizaron pruebas numéricas para tres grados de saturación, a saber, $\mathrm{S}_{r}=0.92,0.97,1.0$ siendo el último caso el correspondiente a suelo totalmente saturado. La tabla 3 muestra los valores empleados.
Tabla 3. Datos de oleaje y del estrato cohesivo en la bahía de Campeche, México

\begin{tabular}{cc}
\hline Período $(T)$ & $12 \mathrm{~s}$ \\
Profundidad $(d)$ & $30 \mathrm{~m}$ \\
Ángulo de incidencia $(\theta)$ & $45^{\circ}$ \\
Relación de Poisson $(\mu)$ & 0.45 \\
Porosidad $\left(n^{\prime}\right)$ & 0.2 \\
Módulo cortante $(G)$ & $10^{8} \mathrm{~N} / \mathrm{m}^{2}$ \\
Permeabilidad $(K)$ & $6 \times 10^{-7} \mathrm{~m} / \mathrm{s}$ \\
Espesor del suelo $(h)$ & $h=0.5 \mathrm{~L}$ \\
Grado de saturación $\left(S_{r}\right)$ & $\mathrm{S}_{\mathrm{r}}=0.92,0.97,1.0$ \\
\hline
\end{tabular}

Con excepción de las distribuciones de los esfuerzos $\tau_{x z}$ y $\tau_{z y}$, los cuales no varían para diferentes condiciones de saturación, se presenta a continuación en las figuras 10 a la 13 los resultados para los esfuerzos y la presión de poro.

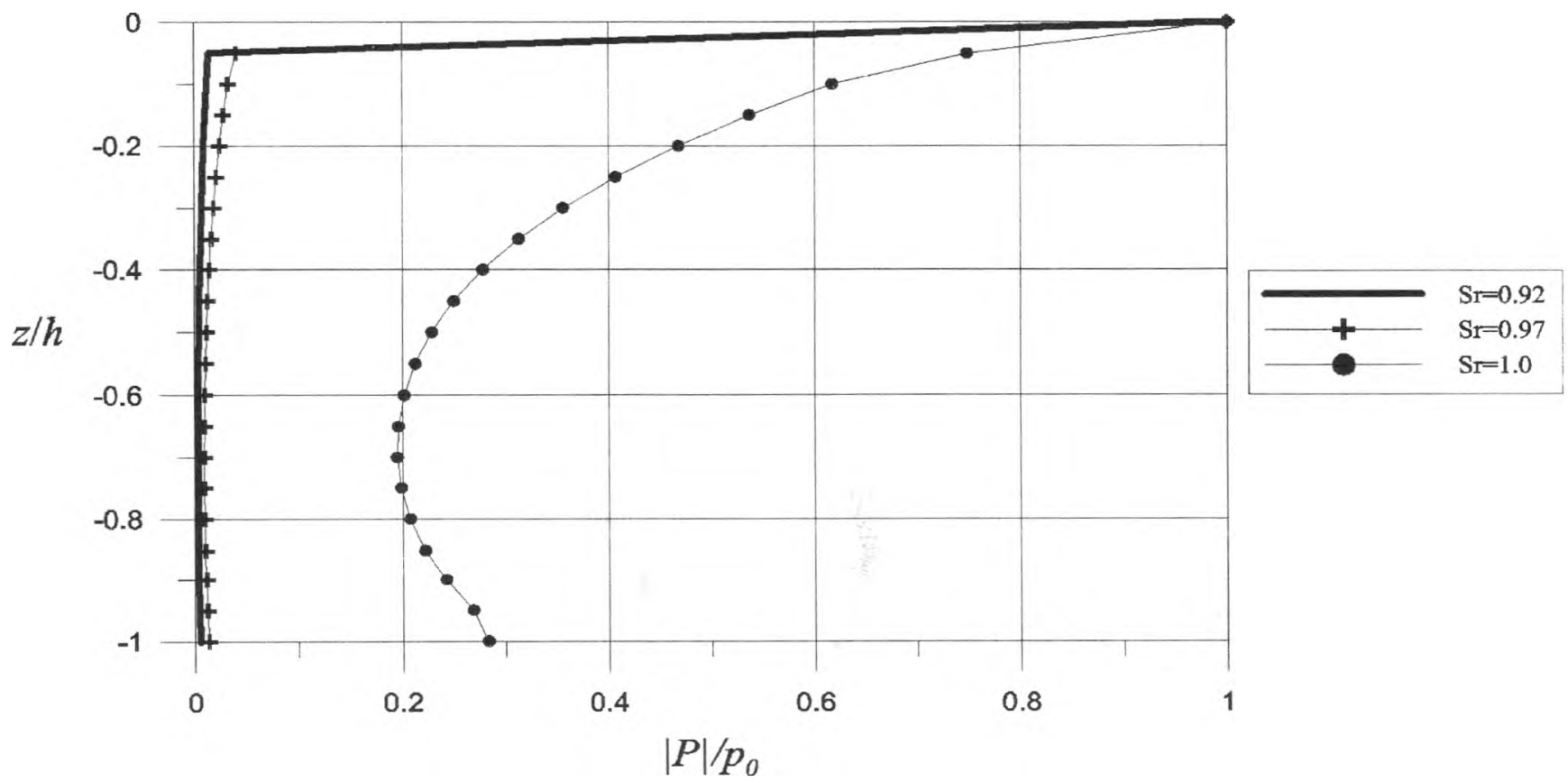

Figura 10. Distribución de la presión de poro $P \mid / p_{0}$ para diferentes grados de saturación y $h=0.5 \mathrm{~L}$ 
E.G. Mendoza-Baldwin, E.E. Salazar-Carrillo y R. Silva-Casarín

DOI:http://dx.doi.org/10.22201/fi.25940732e.2004.05n4.017

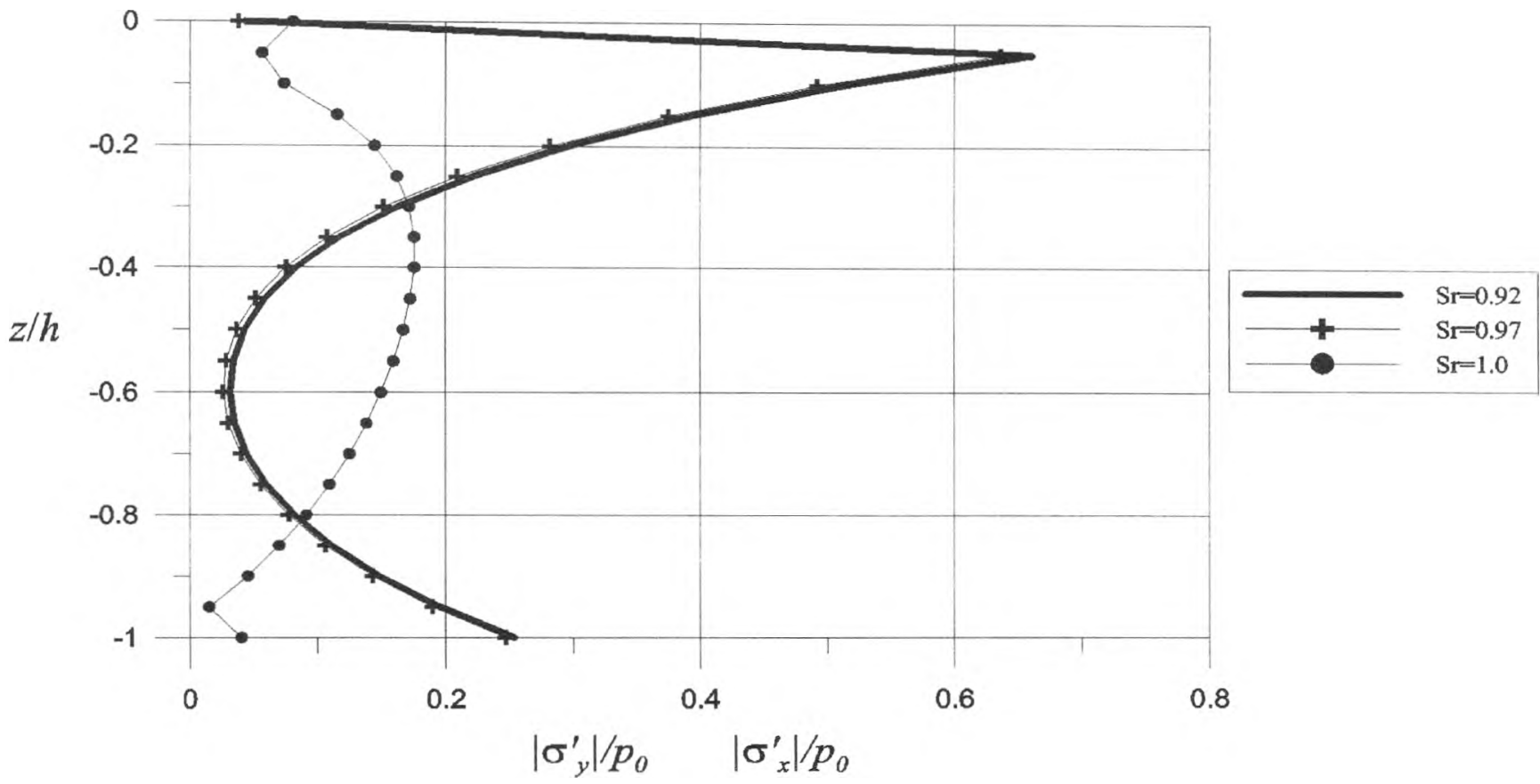

Figura 11. Distribución de los esfuerzos normales $\left|\sigma_{y}^{\prime}\right| / p_{0}$ y $\left|\sigma_{x}^{\prime}\right| / p_{0}$ para diferentes grados de saturación y $h=0.5 \mathrm{~L}$

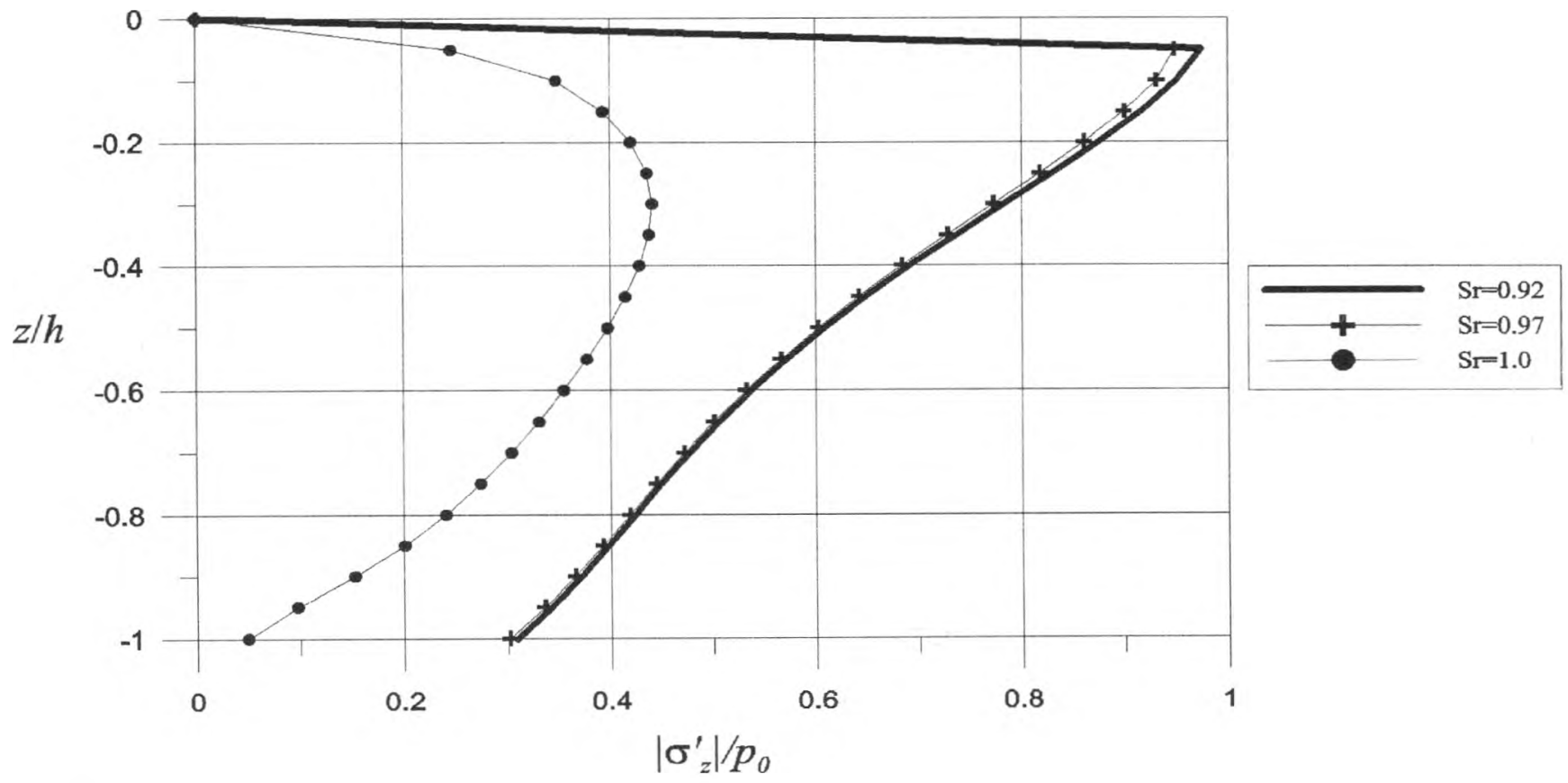

Figura 12. Distribución de los esfuerzos normales $\left|\sigma^{\prime}{ }_{z}\right| / p_{0}$ para diferentes grados de saturación y $h=0.5 L$ 
Acción del oleaje en la estabilidad del lecho marino en la sonda de Campeche. México

DOI:http://dx.doi.org/10.22201/fi.25940732e.2004.05n4.017

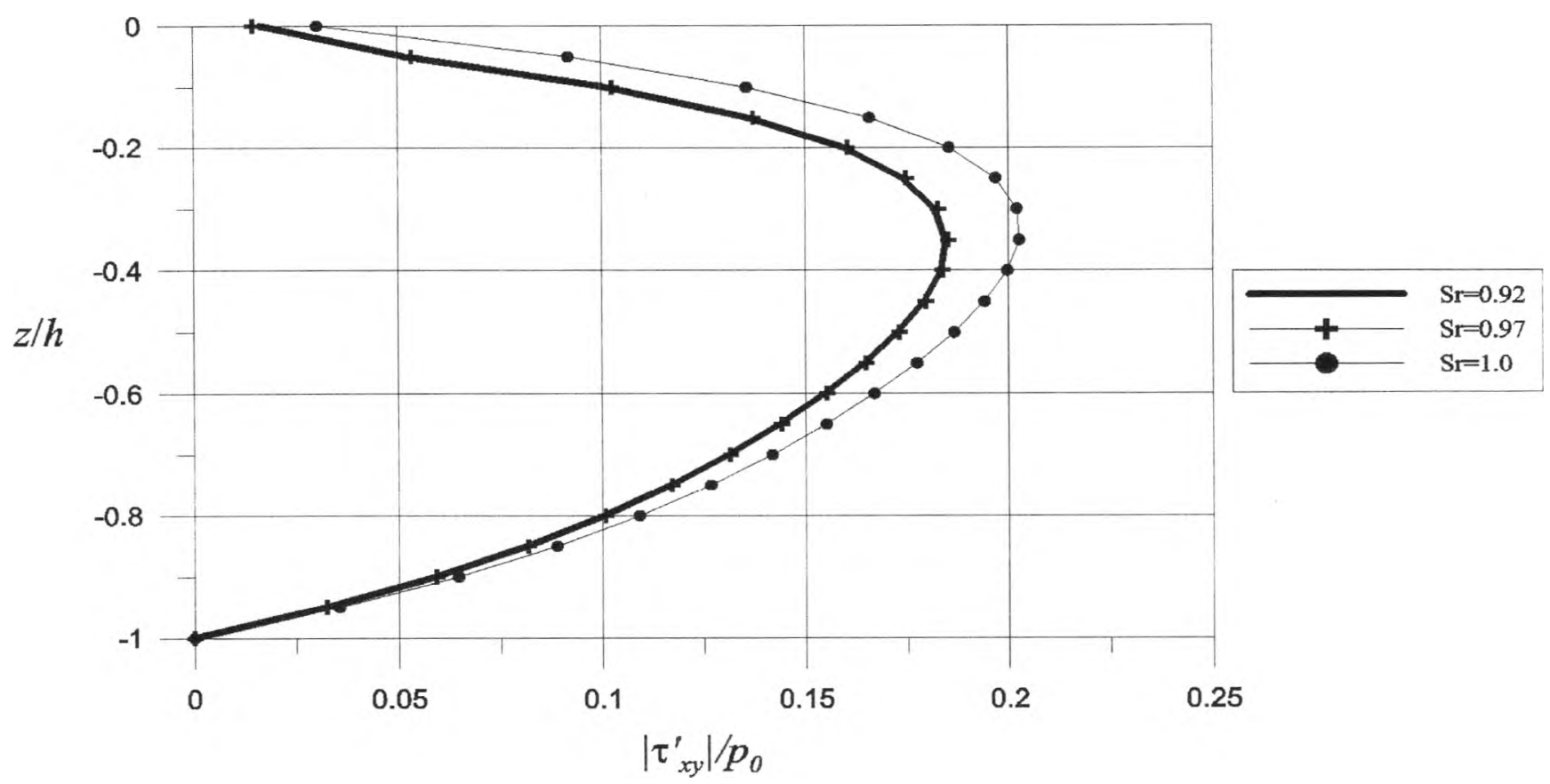

Figura 13. Distribución del esfuerzo cortante $\left|\tau_{x y}\right| / p_{0}$ para diferentes grados de saturación $y \quad h=0.5 \mathrm{~L}$

En las figuras anteriores se observa que la variación entre los grados de saturación no es importante hasta que se alcanza la saturación total, de tal suerte que la decisión de considerar al suelo como totalmente saturado no será relevante, aunque no así el valor del grado de saturación. Finalmente y al igual que en los casos anteriores, es notorio que los esfuerzos máximos se alcanzan muy cerca de la interacción agua-suelo cohesivo.

\section{Estudio de la inestabilidad del fondo marino}

Este estudio consiste en comparar los esfuerzos que resiste el estrato cohesivo contra los esfuerzos inducidos por el oleaje con el fin de detectar las posibles zonas en el perfil vertical de inestabilidad del lecho marino. El conocimiento de la profundidad a la que se presentará la licuefacción inducida, resultado del exceso de esfuerzos, es de vital importancia para el diseño de las líneas de transporte de hidrocarburos.

Los valores del esfuerzo cortante resistente del fondo marino en la sonda de Campeche se obtuvieron de los estudios AKAL-T-J y AKALDB-B cuyos resultados, para la arcilla calcárea local se muestran en el Anexo I. Por lo que respecta a las características del oleaje incidente (estadístico), se tomaron de PEMEX/IMP (2001), considerando condiciones de tormenta, resultando $12.4 \mathrm{~m}$ de altura de ola para 20 metros de profundidad, $14.5 \mathrm{~m}$ para $50 \mathrm{~m}$ de profundidad y $15.5 \mathrm{~m}$ para $100 \mathrm{~m}$ de profundidad.

A continuación, en las figuras 14 a la 16, se muestran los resultados del estudio de inestabilidad para diferentes tirantes de agua (20, 50 y 100 metros). 
E.G. Mendoza-Baldwin, E.E. Salazar-Carrillo y R. Silva-Casarín

DOI:http://dx.doi.org/10.22201/fi.25940732e.2004.05n4.017

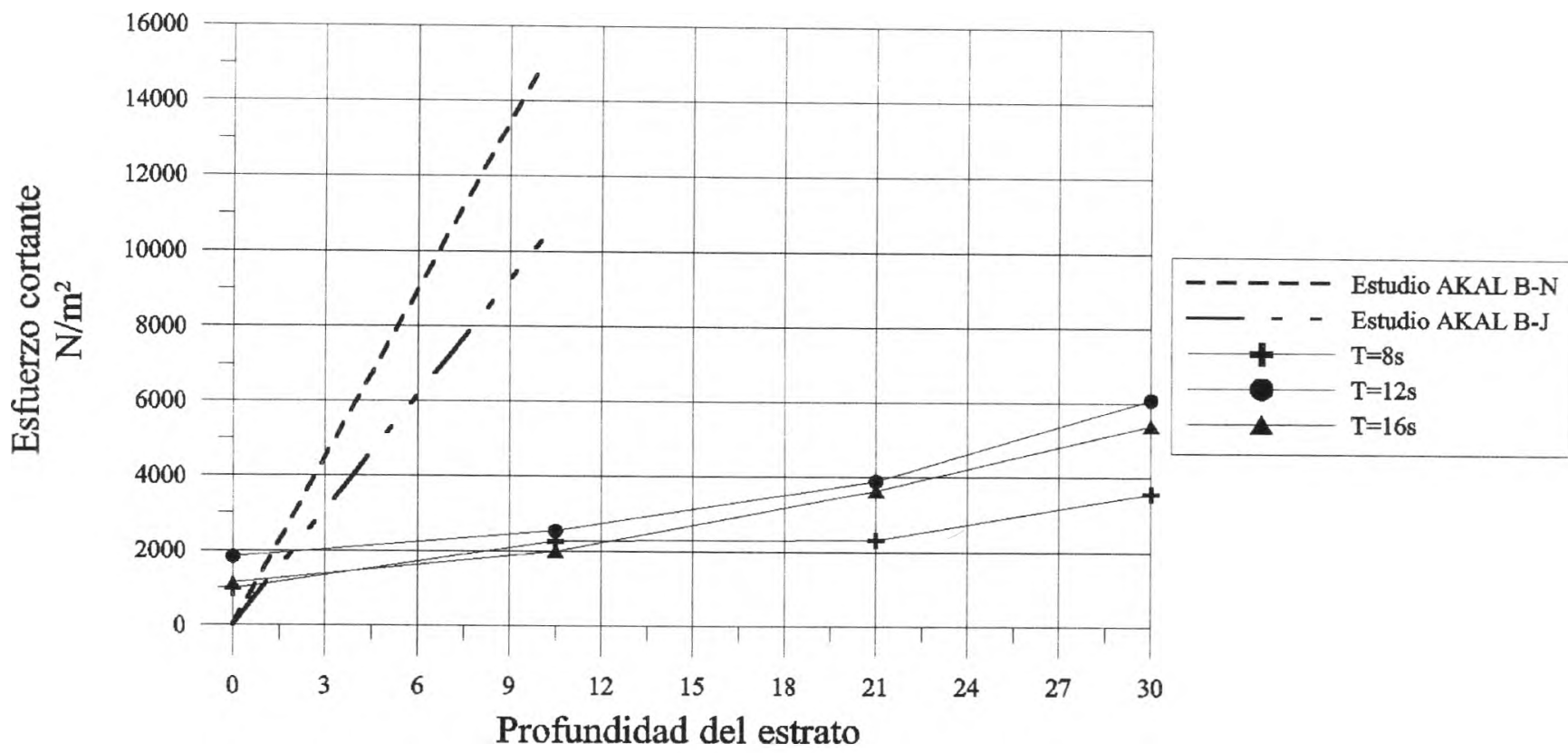

$\mathrm{m}$

Figura 14. Comparación entre los esfuerzos cortantes resistentes e inducidos para tirante de agua de 20 metros

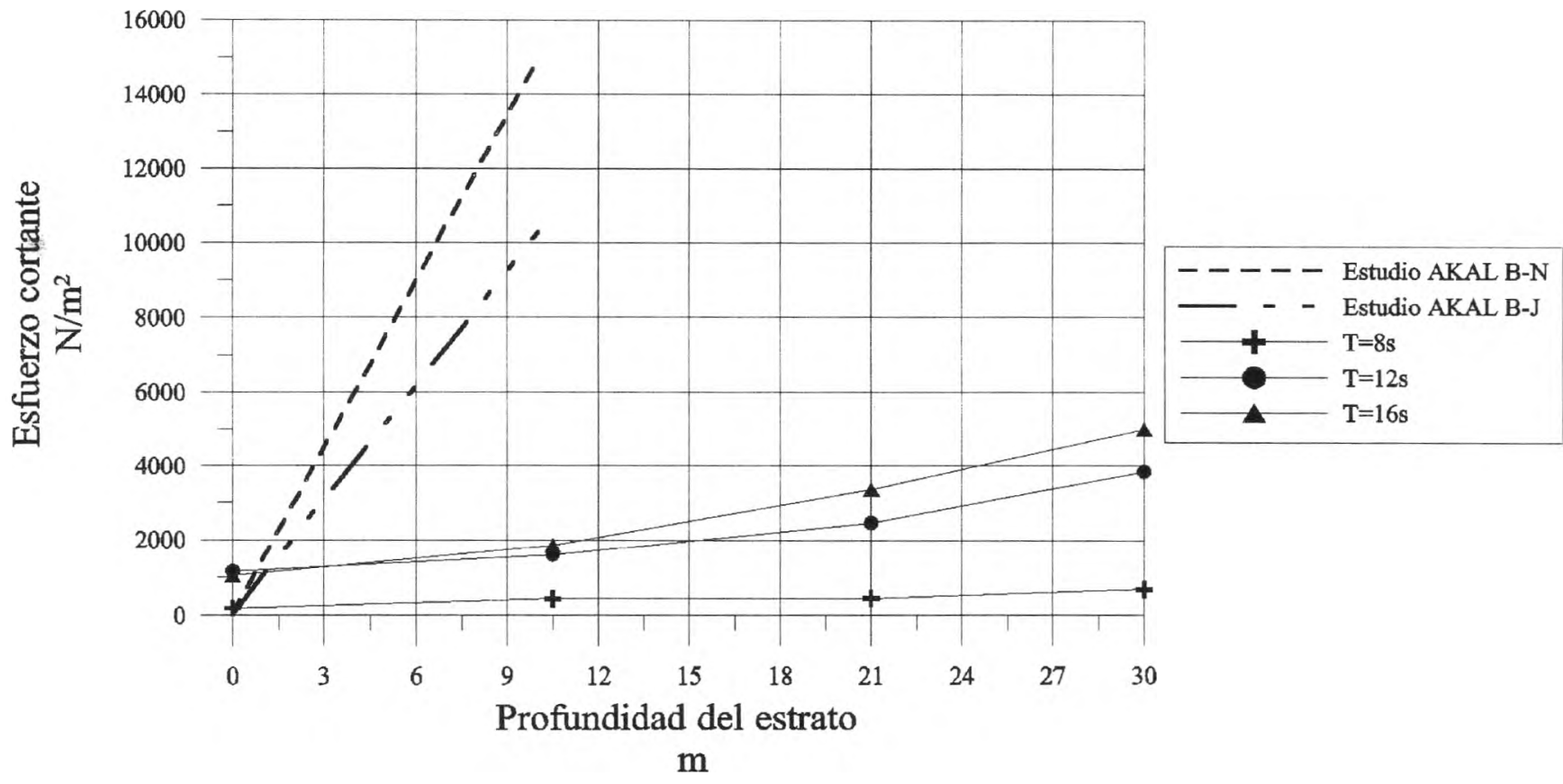

Figura 15. Comparación entre los esfuerzos cortantes resistentes e inducidos para tirante de agua de 50 metros 


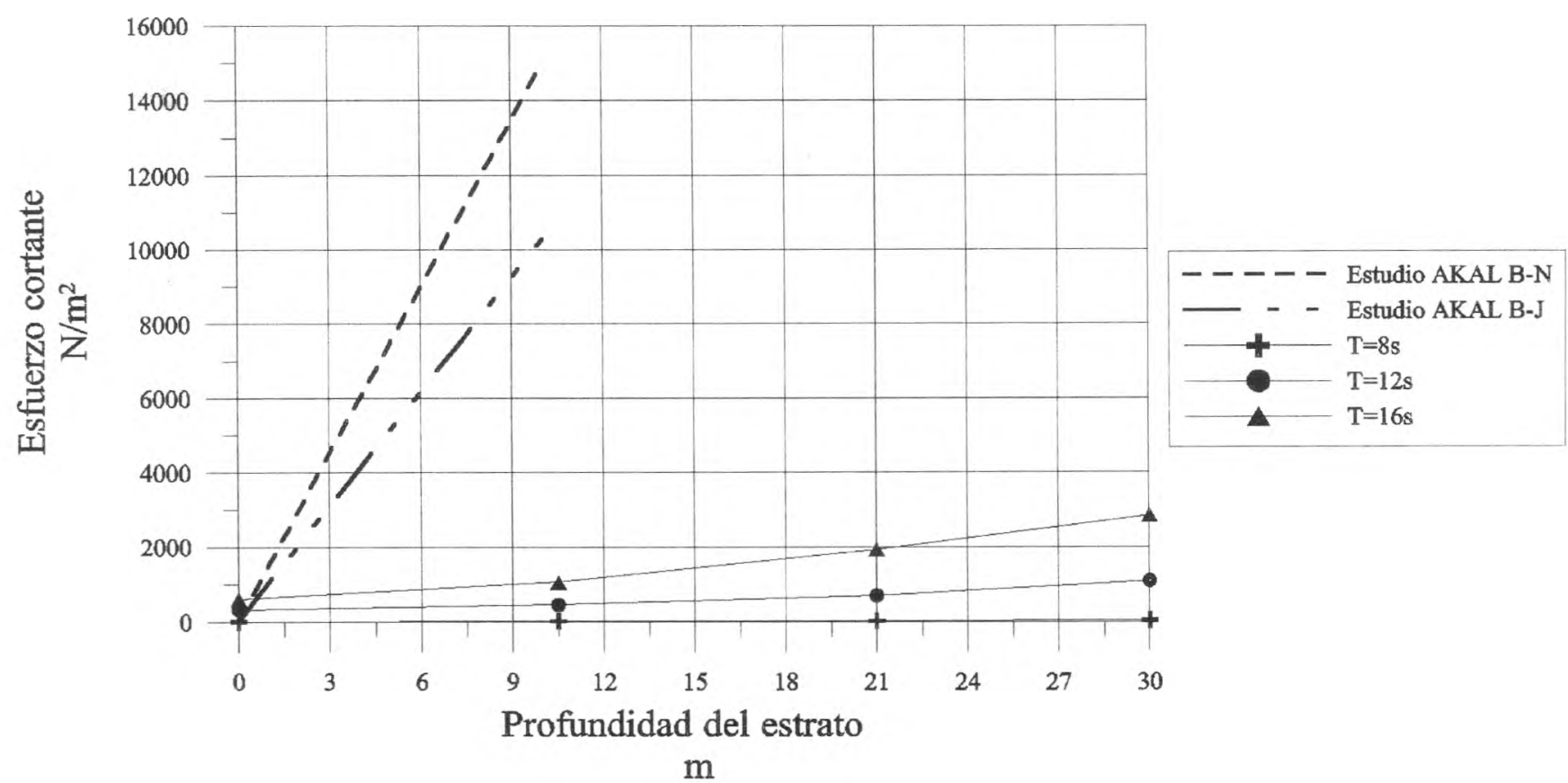

Figura 16. Comparación entre los esfuerzos cortantes resistentes $e$ inducidos para tirante de agua de 100 metros

Tal como se detectó en los resultados del modelo numérico, los mayores esfuerzos se presentan muy cerca de la interacción agua-suelo cohesivo (menos de dos metros de profundidad en todos los casos) siendo ésta la zona en la que se tiene inestabilidad del lecho marino. De este modo, como recomendación inicial, cualquier cimentación, protección o línea de conducción deberá construirse por debajo de dicha zona para asegurar la estabilidad; independientemente de los estudios de socavación y estabilidad de cada estructura.

Finalmente, es de notar que este estudio deberá realizarse para cada localidad y para cada tipo de suelo. Afortunadamente dentro de las capacidades del modelo aquí presentado se cuenta con las de simular, tanto suelos cohesivos como arenosos, así como saturados y no saturados. Por lo que la aplicación de un estudio de inestabilidad se reduce a la consecución de los parámetros de entrada del modelo.

\section{Anexo I}

Estudios geotécnicos en la sonda de Campeche. Figuras 17a, 17b, 18a, 18b, 19a y $19 b$. 
E.G. Mendoza-Baldwin, E.E. Salazar-Carrillo y R. Silva-Casarín

DOI:http://dx.doi.org/10.22201/fi.25940732e.2004.05n4.017

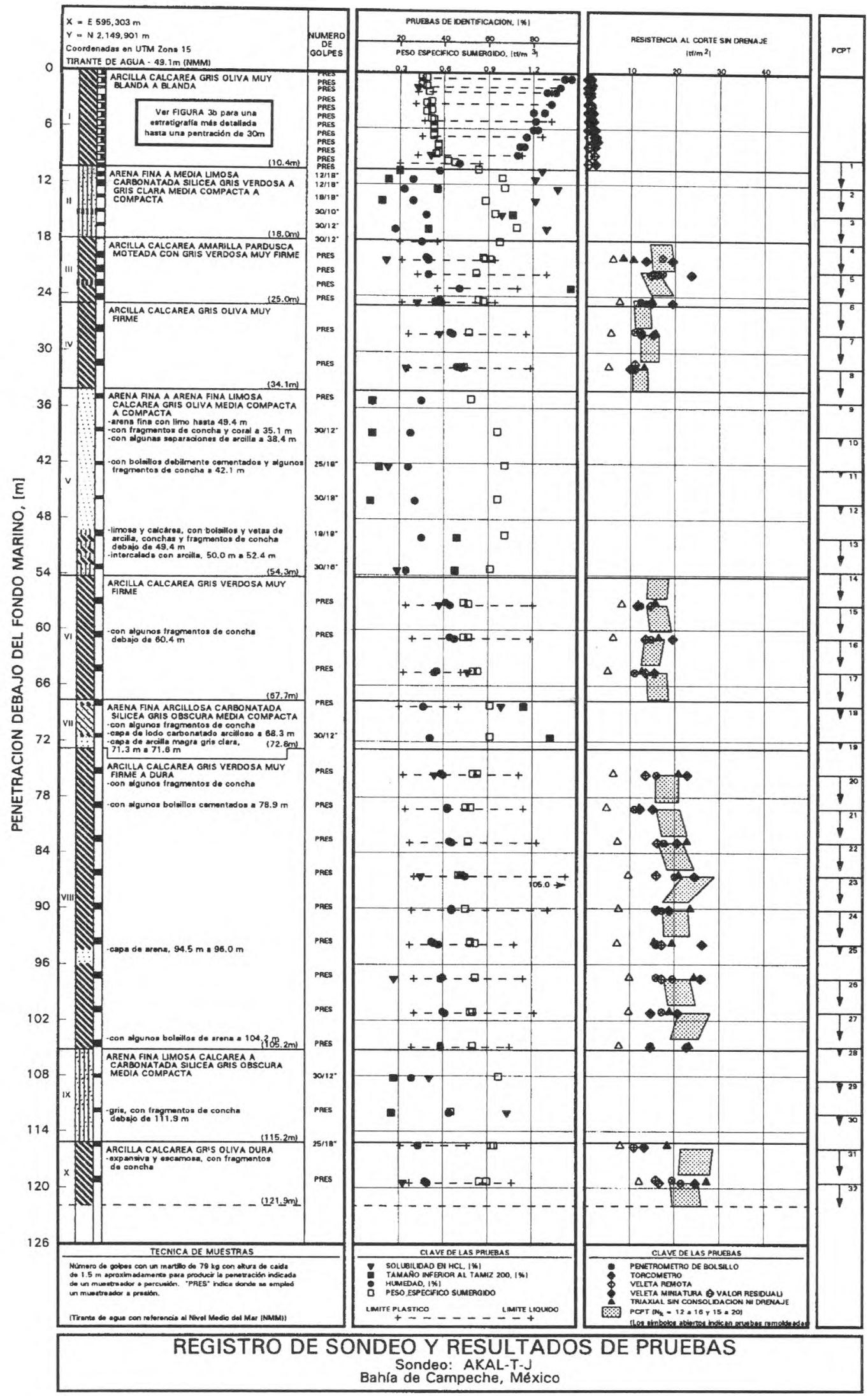

Figura 17 a. Sondeo AKAL-T-J 
Acción del oleaje en la estabilidad del lecho marino en la sonda de Campeche, México DOI:http://dx.doi.org/10.22201/fi.25940732e.2004.05n4.017

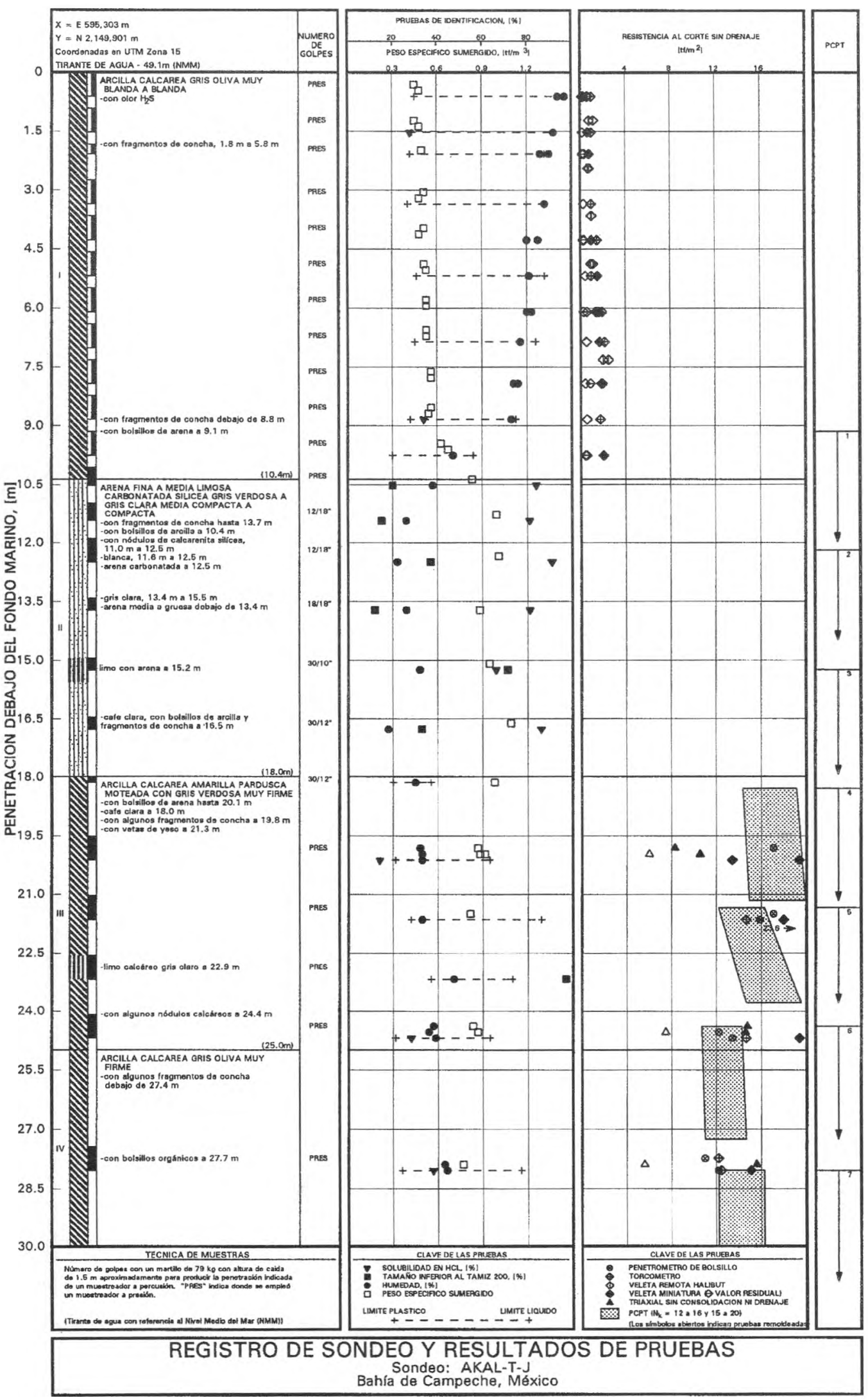

Figura 176. Sondeo AKAL-T-J 
DOI:http://dx.doi.org/10.22201/fi.25940732e.2004.05n4.017
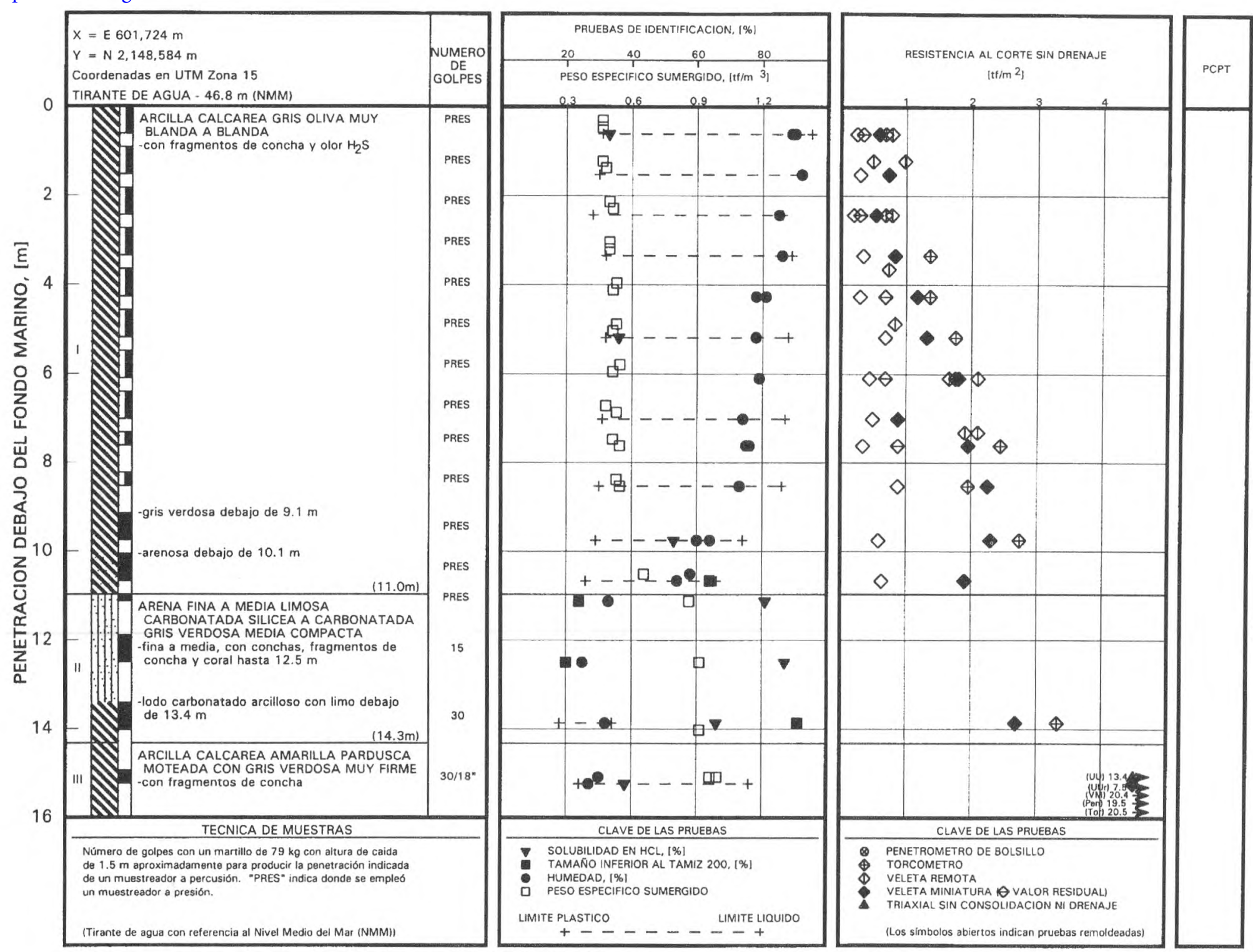

REGISTRO DE SONDEO Y RESULTADOS DE PRUEBAS

Sondeo: AKAL-DB-B

Bahía de Campeche, México 
Acción del oleaje en la estabilidad del lecho marino en la sonda de Campeche, México DOI:http://dx.doi.org/10.22201/fi.25940732e.2004.05n4.017

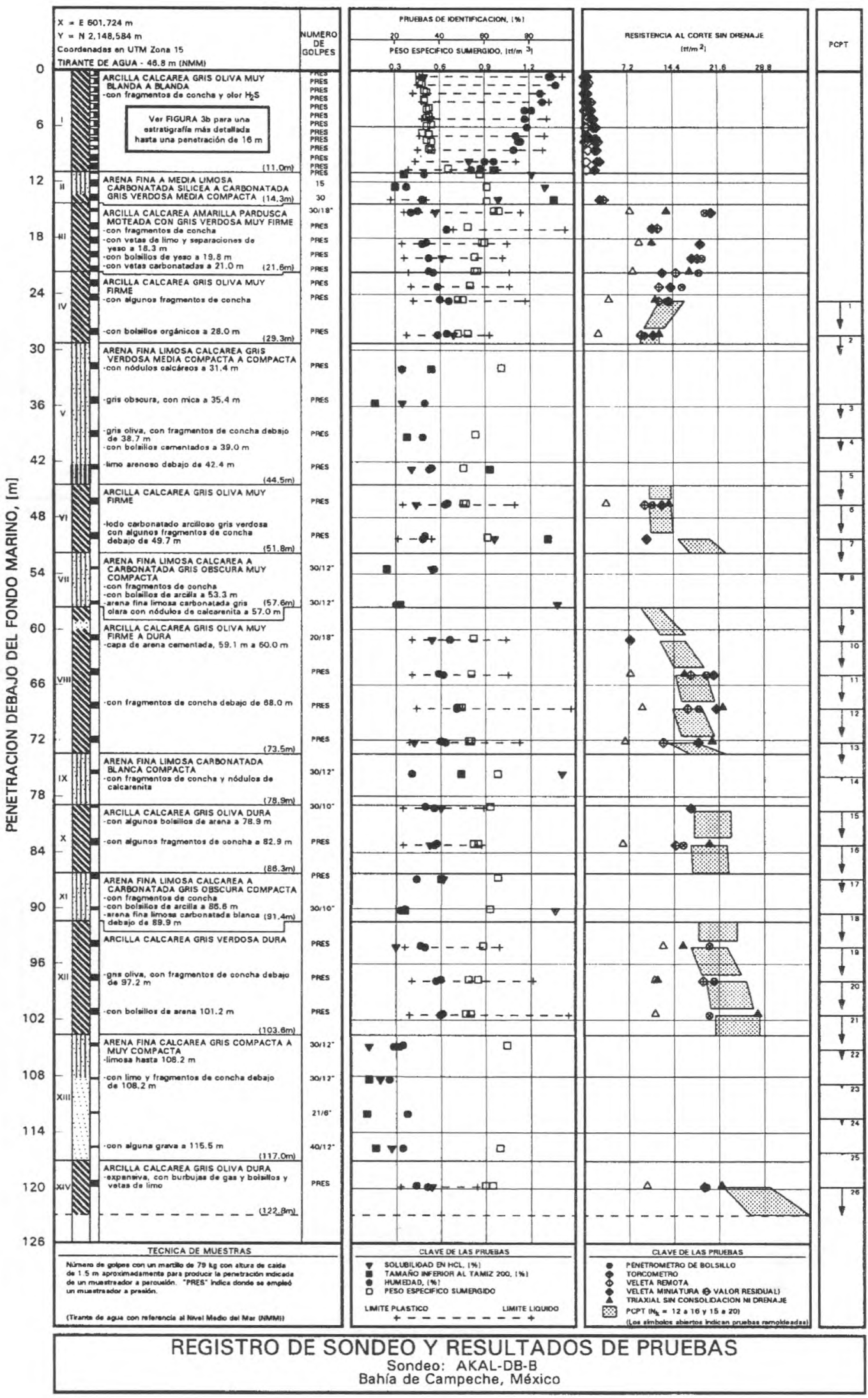

Figura 186. Sondeo AKAL-DB-B 


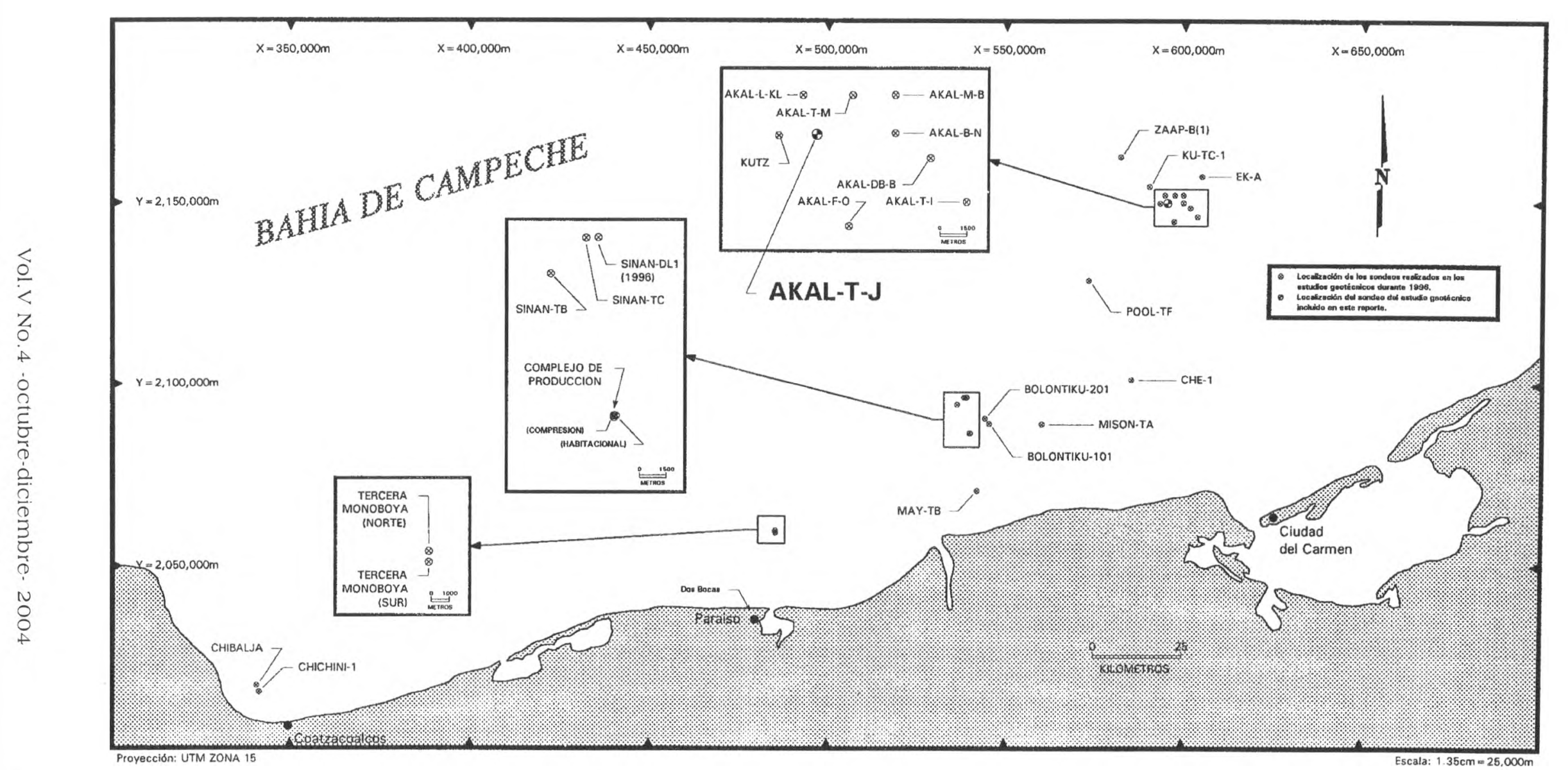




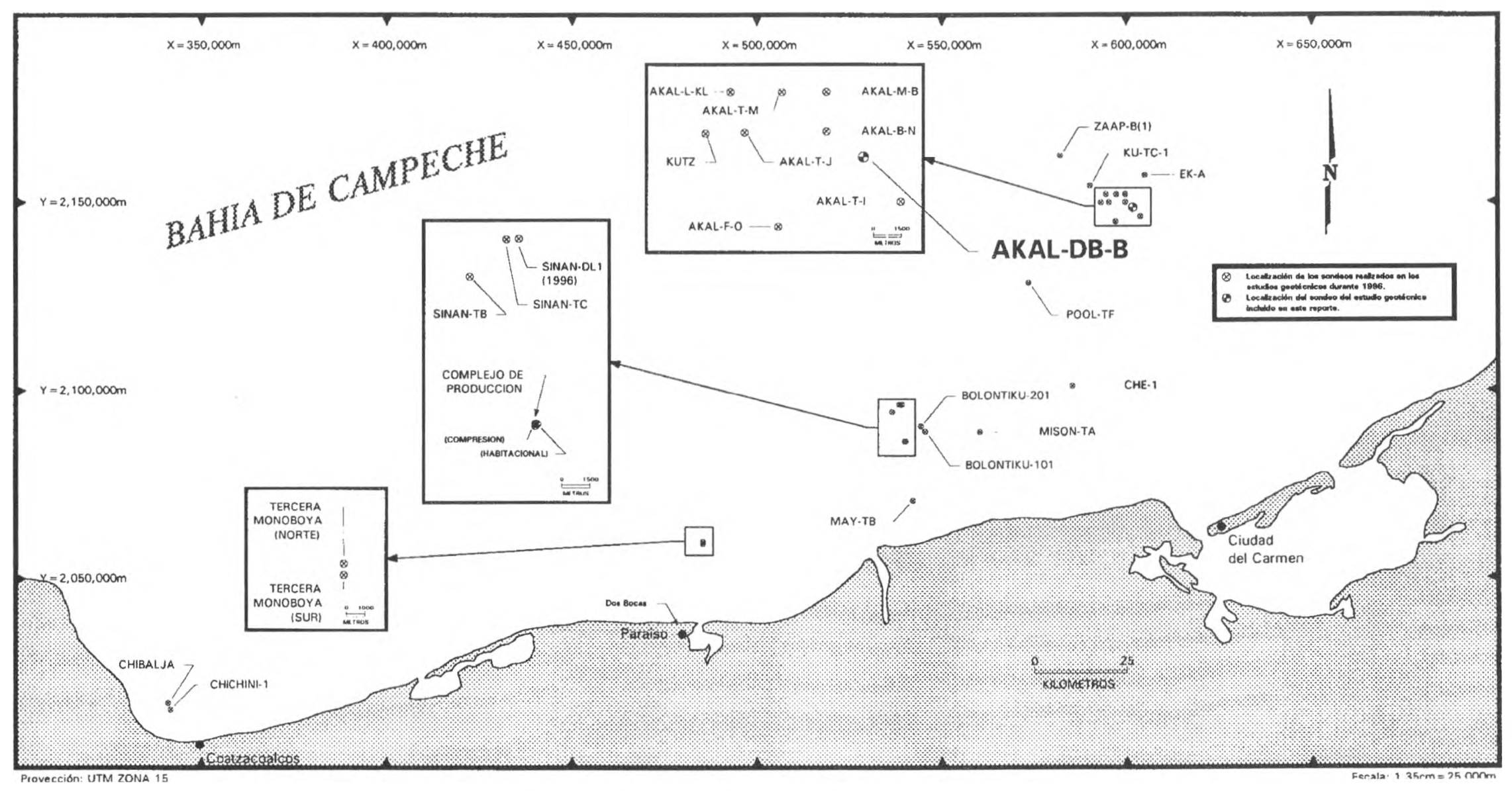

Figura 196. Mapa de localización del sondeo AKAL-DB-B 


\section{Conclusiones}

En este artículo se ha presentado la aplicación de un modelo en tres dimensiones para estimar los esfuerzos y presión de poro inducidos por el oleaje sobre un estrato de suelo poroso de ancho finito como el que se tiene en la Sonda de Campeche, México. El modelo tiene la capacidad de simular diferentes tipos de suelo, grados de saturación, ángulos de incidencia del oleaje y anchuras de estrato poroso.

Adicionalmente al análisis de los resultados numéricos del mismo modelo, su amplitud ha permitido realizar un estudio de inestabilidad del lecho marino en la sonda de Campeche, trabajo del que se puede concluir lo siguiente

Con respecto a los resultados numéricos:

La forma de las distribuciones verticales de esfuerzos y presión tienden a la de una curva similar al aumentar el espesor del estrato cohesivo, de modo que se podría caracterizar su comportamiento para estratos considerablemente anchos.

Los valores máximos de los esfuerzos se presentan muy cerca de la interacción agua-suelo cohesivo.

Para los casos estudiados, el valor del grado de saturación no es importante; sin embargo, el considerar suelo totalmente saturado o no, sí resulta relevante.

Con respecto al estudio de inestabilidad del lecho:
Se encontró que para la sonda de Campeche los esfuerzos inducidos por el oleaje generan una zona de inestabilidad (con la consecuente posibilidad de licuefacción) en los primeros dos metros dentro del estrato cohesivo.

El estudio de inestabilidad resulta un trabajo muy local; no obstante, la aplicación del modelo presentado puede realizarse en una gran variedad de condiciones, tanto del suelo como de oleaje.

\section{Lista de símbolos}

d tirante de agua.

h espesor del estrato cohesivo.

k. número de onda.

$k_{x}, k_{y}$ componentes vectoriales del número de onda.

$m, n$ componentes trigonométricas del número de onda.

n' porosidad del suelo.

$p_{0} \quad$ amplitud de la presión inducida.

$t$ tiempo.

$x, y, z$ ejes del sistema coordenado.

$B_{i} \quad$ subcoeficientes para el cálculo de los $\mathrm{C}_{i}$.

$C_{i} \quad$ coeficientes.

$D_{i} \quad$ subcoeficientes para el calculo de los $\mathrm{C}_{i}$.

E módulo de Young.

G módulo de cortante del suelo.

$H$ altura de ola.

$\bar{K} \quad$ vector de coeficiente de permeabilidad del suelo.

$K^{\prime} \quad$ módulo de masa aparente del agua en los poros.

Kw módulo de masa real de elasticidad del agua. 
Acción del oleaje en la estabilidad del lecho marino en la sonda de Campeche, México DOI:http://dx.doi.org/10.22201/fi.25940732e.2004.05n4.017

$K_{x} K_{\psi} K_{z}$ coeficientes de permeabilidad del suelo en las direcciones $x, y, z$.

L longitud de onda.

$L_{x y} L_{y}$ componentes de la longitud de onda.

$P \quad$ presión de poro inducida.

$P_{w o} \quad$ presión hidrostática ejercida por el agua.

$P_{1}, P_{2}$ componentes de la presión inducida por el oleaje incidente y reflejado.

$\mathrm{Sr} \quad$ grado de saturación.

$\beta \quad$ compresibilidad del fluido en los poros.

$\delta$ parámetro combinado del oleaje y el suelo.

$\varepsilon \quad$ volumen de esfuerzos del suelo.

$\gamma_{\mathrm{w}} \quad$ peso específico del agua en los poros.

$\lambda$ parámetro adimensional relativo a la anisotropía y el grado de saturación del suelo.

$\mu \quad$ relación de Poisson.

$\theta$ ángulo de incidencia del oleaje.

$\sigma^{\prime}{ }_{i} \quad$ componentes del esfuerzo efectivo normal.

$\tau_{i j}^{\prime} \quad$ componentes del esfuerzo cortante efectivo.

$\omega \quad$ frecuencia angular del oleaje.

$\xi, \zeta, \chi$ desplazamientos del suelo en las direcciones $x, y, z$.

\section{Referencias}

Biot M. (1941). General Theory of Three Dimensional Consolidation. Journal of Applied Physics, Vol. 12, pp. 155-164.

Bowles J. (1997). Foundation Analysis and Design. Mc Graw Hill, EUA.

Dawson T. (1978). Wave Propagation Over a Deformable Bed Floor. Ocean Engineering, Vol. 5, pp. 227-234.

Gatmiri B. (1990). A Simplified Finite Element Analysis of Wave Induced
Effective Stress and Pore Pressures in Permeable Sea Beds. Géotechnique, Vol. 40 , pp. 15-30.

Hsu J. y Jeng D. (1994). Wave-induced Soil Response in an Unsaturated Anisotropic Seabed of Finite Thickness. International Journal for Numerical Methods and Analytical Methods in Geomechanics, Vol. 18, pp. 785-807.

Hsu J., Jeng D. y Tsai C. (1993). Short-Crested Wave-Induced Soil Response in a Porous Seabed of Infinite Thickness. International Journal for Numerical Methods and Analytical Methods in Geomechanics, Vol. 19, 825-849.

Jeng D. (1997). Wave-Induced Seabed Response in Front of a Breakwater. Tesis Doctoral, University of Western Australia, Crawley, Australia.

Liu P. (1977). On Gravity Waves Propagated Over a Layered Permeable Bed. Coastal Engineering, Vol .1, pp. 135-148. Liu P. y Dalrymple L. (1984). The Damping of Gravity Water-Waves Due to Percolation. Coastal Engineering, Vol.8, pp. 33-49.

Madsen O. (1978). Wave Induced Pore Pressures and Effective Stresses in a Porous Bed. Géotechnique, Vol. 28, pp. 377-393.

Mallard W. y Dalrymple R.A. (1977). Water Waves Ppropagating Over a Deformable Bottom. 9th Annual Offshore Technology Conference. Houston.

Mei C. y Foda M. (1981). Wave Induced Response in a Fluid-Filled Poro-Elastic Solid with a Free Surface - a Boundary Layer Theory. Geophysical Journal of the Royal Astronomical Society, Vol. 66, pp. 597-631.

Moshagen H. y Torum A. (1975). Wave Induced Pressures in Permeable Seabed. Journal of Waterways, Harbours and Coastal Engineering, Vol. 101. 
Nakamura H., Onishi R. y Minamide H. (1973). On the Seepage in the Seabed Due to Waves. 20th. Coastal Engineering Conference. Japón.

PEMEX/IMP (2001). Criterio transitorio para el diseño y la evaluación de líneas submarinas en la sonda de Campeche. Reporte No. 0201-2927-16, Petróleos Mexicanos / Instituto Mexicano del Petróleo.

Prevost J. y Hughes J. (1980). Finite Element Solution of Boundary Value Problems in Soil Mechanics. International Symposium on Soils Under Cyclic and Transient Loading. Rotterdam.

Putnam J. (1949). Loss of Wave Energy due to Percolation in a Permeable Sea Bottom. Trans. Am. Geophys. Union.
Salazar E. (2001). Cinemática del oleaje en suelos disipativos. Tesis de maestría, Universidad Nacional Autónoma de México, México, DF.

Yamamoto T. (1977). Wave Induced Instability Sea Beds. Coastal Sediments ' 77. Charleston, SC.

Yamamoto T. (1983). On the Response of a Coulomb-Damped Poro-Plastic Bed to Water Waves. Marine Geotechnology, Vol. 5, pp. 93-130.

Yamamoto T., Koning H., Sellmejjer H. y Hijum E. (1978). On the Response of a Poroelastic Bed to Water Waves. Journal of Fluid Mechanics, Vol. 87, pp. 193-206.

\section{Semblanza de los autores}

Edgar Gerardo Mendoza-Baldwin. Obtuvo el título de ingeniero civil en el año 2000 y el grado de maestro en ingeniería civil (hidráulica) en el 2002, ambos en la División de Estudios de Posgrado de la Facultad de Ingeniería, UNAM. Actualmente es estudiante de doctorado y becario del Instituto de Ingeniería, así como profesor de asignatura de la División de Ciencias Básicas de la misma Facultad.

Edgar Eduardo Salazar-Carrillo. Se graduó como ingeniero civil en el Instituto Politécnico Nacional de la Ciudad de México. Posteriormente, realizó su maestría en el área de ingeniería hidráulica en la UNAM, en donde elaboró estudios sobre la estabilidad del suelo marino. Ahora, labora en el Instituto Mexicano del Petróleo dentro del programa de desarrollo tecnológico en aguas profundas.

Rodolfo Silva-Casarín. Egresado como ingeniero civil de la ENEP-Acatlán en 1988, obtuvo su maestría en administración de empresas en 1990 en la Universidad de Chapultepec. Es maestro en ingeniería oceanográfica (1992) y doctor en ingeniería de caminos canales puertos de la Universidad de Cantabria, España (1995). A la fecha es investigador titular del Instituto de Ingeniería y profesor de la División de Estudios de Posgrado de la Facultad de Ingeniería, UNAM. 
DOI:http://dx.doi.org/10.22201/fi.25940732e.2004.05n4.017 